\title{
Applications des impulsions lasers ultra-brèves
}

\author{
D. Hulin
}

Laboratoire d'Optique Appliquée, E.N.S.T.A., Ecole Polytechnique, URA 1406 du CNRS, Centre de l'Yvette, 91120 Palaiseau, France

\section{INTRODUCTION}

La production et surtout la fiabilisation des lasers à impulsions femtosecondes ont ouvert les portes d'un monde fascinant à plus d'un titre.

Le domaine temporel tout d'abord. Il est évident que tout phénomène peut être regardé en super-ralenti, comme avec un super-stroboscope. On observe alors des durées de vie courtes, des phénomènes cohérents, les premières étapes d'une relaxation, d'un changement de phase ou d'une réaction. Dans les systèmes complexes, on a enfin les clés qui permettent de comprendre les mécanismes fondamentaux, car à cette échelle de temps là, tous les processus secondaires sont comme gelés et seules sont actives les étapes primordiales. Enfin cela permet d'étudier la physique de systèmes hors d'équilibre, avec des aspects très nouveaux par rapport aux systèmes relaxés ou moyennés.

L'optique non-linéaire bien sûr ensuite. Grâce à la faible durée des impulsions, toute énergie même modeste donne lieu à des puissances colossales, qui correctement focalisées dépassent déjà $10^{18} \mathrm{Wcm}^{-2}$. Il est presque magique de jouer avec les longueurs d'onde en utilisant l'automodulation de phase, les effets paramétriques, les additions ou soustractions de fréquences, .... Toute étude du type " pompe et test " n'est aussi qu'une expérience d'optique non-linéaire où deux faisceaux interagissent via une modification de la matière.

Le domaine des fortes intensités et temps courts. Depuis quelques années, les impulsions femtosecondes intenses ont montré des caractéristiques particulières qui donnent des résultats se distinguant nettement de ceux obtenus aussi à forte intensité mais en des temps plus longs. C'est actuellement une voie pleine de promesses, abordant le domaine des phénomènes relativistes.

Les temps de quelques femtosecondes $\left(1 \mathrm{fs}=10^{-15_{\mathrm{s}}}\right.$ ) ne sont pas facilement accessibles à notre imagination. On se les représente mieux en les transformant en distances. A la vitesse de $300000 \mathrm{~km} / \mathrm{s}$, la lumière parcourt environ la distance terre-lune en une seconde alors qu'elle parcourt $3 \mathrm{~mm}$ en $10 \mathrm{fs}$. Des transitoires entre la picoseconde et la femtoseconde seront décrits à l'aide de fréquences allant du térahertz $\left(10^{12} \mathrm{~Hz}\right)$ au pétahertz $\left(10^{15} \mathrm{~Hz}\right)$.

De très grandes possibilités d'études sont donc ouvertes par la maîtrise des impulsions lasers femtosecondes. Aussi il n'est pas étonnant que Nicolas Bloembergen, un récent prix Nobel de physique, ait déclaré pour un sondage que, si il devait recommencer aujourd'hui une vie scientifique en optique, il choisirait le domaine des femtosecondes. 


\section{LES IMPULSIONS FEMTOSECONDES}

Il n'est pas inutile de revenir rapidement sur quelques aspects des impulsions lasers femtosecondes, bien que l'essentiel en ait été donné dans le chapitre "Sources laser femtosecondes" par A. Brun et P. Georges dans ce livre.

Une impulsion dite gaussienne est constituée à partir de l'oscillation du champ électromagnétique à la fréquence $\omega_{0}$, dite fréquence porteuse, multipliée par une fonction enveloppe de type gaussien. Le champ électrique en fonction du temps t s'écrit:

$$
E(t)=E_{0} \exp \left(-t^{2} / 2 \tau^{2}\right) \cos \left(\omega_{0} t\right)
$$

où $\tau$ est un paramètre relié à la durée de l'impulsion. L'enveloppe de l'intensité associée a pour expression :

$$
I(t)=I_{0} \exp \left(-t^{2} / \tau^{2}\right)
$$

Cette impulsion a pour largeur à mi-hauteur $\Delta \mathrm{t}=\tau \times 2 \sqrt{\mathrm{L}} \operatorname{og} 2=\tau / 0.6$. L'impulsion la plus courte produite et utilisée est de 6 fs (Fork et al., 1987). Dans le visible, cela ne représente qu'environ 6 oscillations du champ électromagnétique.

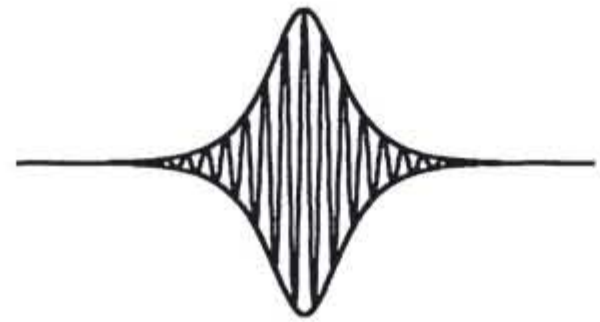

$\Delta t=10 \mathrm{fs}$

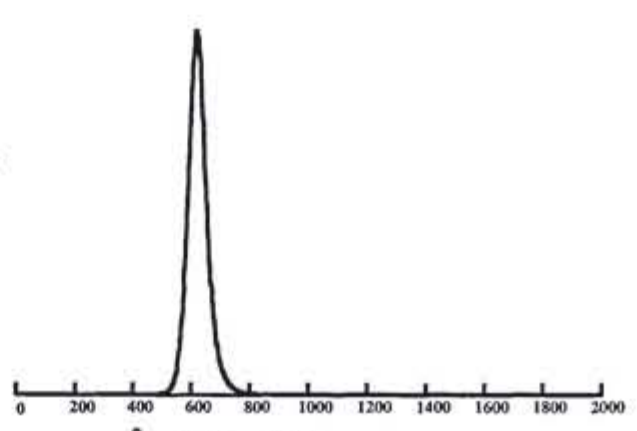

$\lambda$ en $\mathbf{n m}$

Figure 1 : Forme temporelle et spectre d'une impulsion de 10 femtosecondes.

On voit qu'une fréquence porteuse peut encore être définie, mais l'approximation de l'enveloppe lentement variable est à la limite de validité. Le spectre (figure 1) d'une impulsion de $10 \mathrm{fs}$, obtenu par transformée de Fourier, montre une certaine largeur $\Delta v$ autour de la fréquence centrale. Dans la relation $\Delta \mathrm{t} \times \Delta \mathrm{v} \geq \mathrm{a}$, (a voisin de 1), le coefficient "a" dépend de la forme temporelle exacte de l'impulsion (gaussienne, bi-exponentielle, sécante hyperbolique carrée, ...). L'égalité n'est atteinte que pour une impulsion dont la durée et le spectre se correspondent par transformée de Fourier. Dans ce cas, pour une largeur spectrale donnée, on a alors la plus courte durée possible.

Une impulsion de durée 1 fs centrée dans le visible ne comporte plus qu'une oscillation du champ électromagnétique. Son -spectre possède des composantes dans l'ultraviolet et linfrarouge : elle est plus que blanche.

Une telle impulsion n'a jamais été produite et, sauf si elle se propageait dans le vide, elle serait très difficile à garder aussi courte lors de sa propagation à cause des différentes vitesses selon les longueurs d'onde. La manipulation d'une impulsion de $10 \mathrm{fs}$ est déjà délicate sachant 
que la traversée de $1 \mathrm{~mm}$ de verre double presque sa durée. Les systèmes correcteurs à dispersion négative (prismes ou réseaux) rendent possibles la maîtrise de ces temps courts.
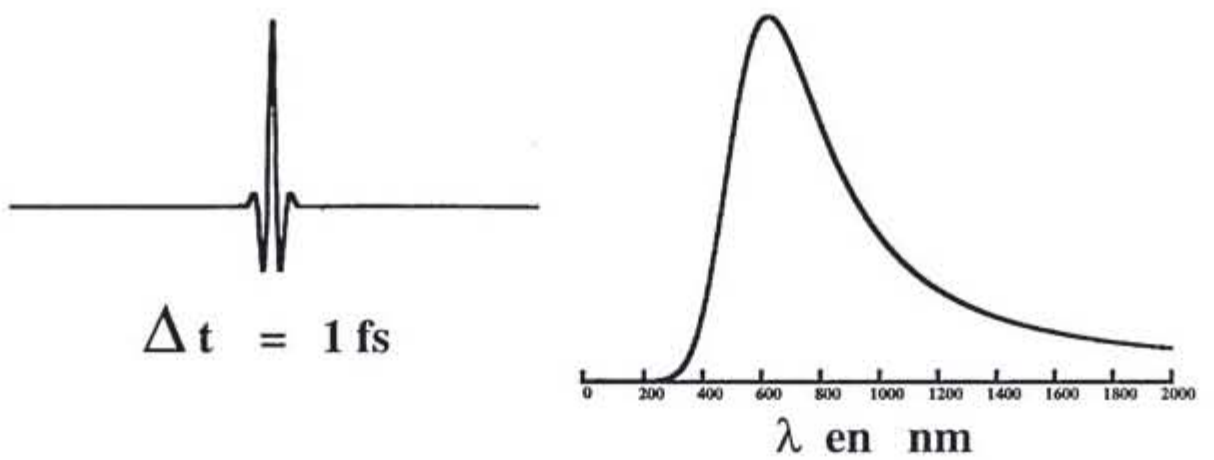

Figure 2 : Profil temporel et spectre d'une impulsion de $1 \mathrm{fs}$.

\section{PRINCIPE DES MESURES RÉSOLUES EN TEMPS}

Il ne faut pas espérer utiliser un système électronique pour mesurer un phénomène à de telles échelles de temps. La plupart des mesures aux temps courts utilisent le principe de la méthode "pompe et test" ou "pompe et sonde", c'est-à-dire l'utilisation de deux impulsions lasers.

\subsection{Méthode pompe et test}

Une première impulsion lumineuse, appelée pompe, déclenche un événement au temps to pris souvent comme origine des temps $\left(t_{0}=0\right)$. Le système évolue ensuite après cette excitation. Cette perturbation se traduit par un changement d'au moins une des caractéristiques du système. Supposons d'abord que cela modifie une de ses caractéristiques optiques (absorption ou réflectivité). Le signal correspondant passe de sa valeur au repos $S(-\infty)$ à $S(t)=S\left(t_{0}+\Delta t\right), \Delta t$ étant le délai entre le début de la perturbation (arrivée de la première impulsion) et l'instant $t$. Si la mesure est faite au temps $t$, on recueille la valeur $S(t)$ (figure 3 ). Cette mesure est faite par une deuxième impulsion lumineuse, appelée test ou sonde, qui est censée être suffisamment peu intense pour ne pas perturber à nouveau le système.

Cette mesure ne donne qu'un point du signal $S(t)$. Cependant, si on recommence tout le processus en changeant le délai $\Delta t$, on aura un autre point de la courbe $S(t)$. Pour explorer toute la courbe, il faut prendre de nombreuses valeurs de $\Delta t$. Pour un délai négatif, la mesure donne la valeur du signal non perturbé. Pour un délai très grand, le signal tend à nouveau vers sa valeur au repos. Il faut d'ailleurs que le temps entre deux excitations soit plus long que le temps de retour au repos si on veut mesurer le même système à chaque fois. 


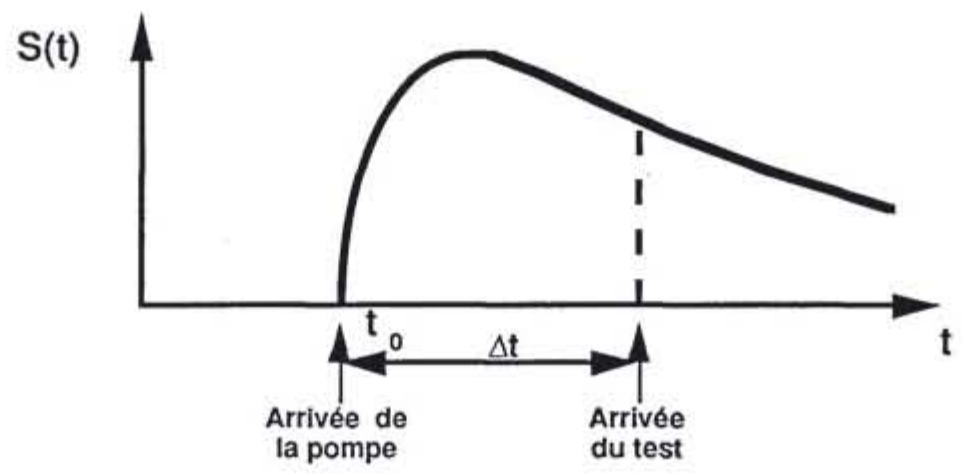

Figure 3 : Schéma de principe d'une mesure pompe-test.

L'impulsion de pompe et l'impulsion de test pourraient venir de lasers différents. Toutefois il est difficile de synchroniser deux lasers avec une très bonne précision et ceci introduit une incertitude sur la valeur de $\Delta t$. Pour éviter cela, les impulsions de pompe et de test sont issues de la même impulsion partagée en deux par un miroir semi-réfléchissant. Après leur séparation, les deux impulsions parcourent des trajets optiques différents qui ont une longueur égale pour $\Delta t=0$. Il suffit ensuite de faire varier la longueur d'un des trajets pour pouvoir explorer toute la courbe $S(\Delta t)$. Les différences de temps d'arrivée sont transformées en différences de longueurs qui, elles, sont mesurables avec une précision meilleure que le micromètre ( $1 \mu \mathrm{m}=3.3 \mathrm{fs}$ ). En pratique, le délai nul est trouvé grâce à un phénomène physique qui ne se produit que pour le recouvrement temporel et spatial des deux impulsions et ce sont les variations par rapport à cette position qui sont mesurées.

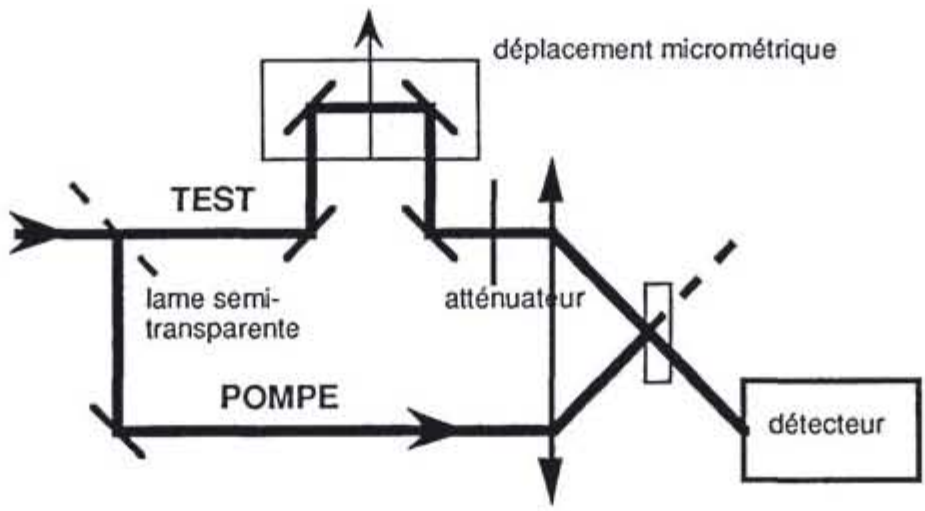

Figure 4: Schéma d'une expérience d'absorption résolue en temps

Le détecteur recueille le faisceau de test qui a traversé l'échantillon. A ce niveau-là, il n'est plus du tout question de résolution temporelle, tout l'aspect discrimination en temps venant du recouvrement ou du délai entre les deux impulsions au niveau de l'échantillon où elles se 
croisent spatialement. En particulier, le détecteur peut intégrer et accumuler le signal de test. On utilise souvent une barrette de diodes précédée d'un spectromètre.

\subsection{Le faisceau de test}

Une des caractéristiques du test est d'être peu intense pour ne pas perturber l'objet à étudier (présence de l'atténuateur sur le trajet du faisceau test). Toutefois, dans le schéma ci-dessus, le test est à la même longueur d'onde que le faisceau de pompe. Or la signature optique de la perturbation, la modification d'une résonance ou l'apparition d'une nouvelle bande d'absorption par exemple, peut se trouver à une autre longueur d'onde que celle de la perturbation. Pour obtenir un test ayant de nombreuses composantes spectrales, on utilise l'automodulation de phase.

Une impulsion intense et brève subit de l'automodulation de phase dès qu'elle traverse un milieu matériel et en particulier un milieu transparent. Celui utilisé pour la génération d'un test spectralement large est souvent de l'eau pour des raisons de simplicité ou une fibre optique pour allonger la longueur d'interaction lorsque l'intensité initiale n'est pas très intense. Le principe en est simple: la fréquence d'une onde électromagnétique est la dérivée par rapport au temps de la phase $\phi$ :

$$
\phi=\left(\omega_{0} \mathrm{t}-\mathrm{kz}\right)=\left(\omega_{0} \mathrm{t}-\omega_{0} \mathrm{z} \mathrm{n} / \mathrm{c}\right)
$$

Par ailleurs, l'indice $\mathrm{n}$ a toujours une composante non-linéaire qui dépend de l'intensité :

$$
\mathrm{n}=\mathrm{n}_{0}+\mathrm{n}_{2} \mathrm{I}
$$

Cette dernière composante n'est effective que lorsque l'intensité I est très grande car $\mathrm{n}_{2}$ est très petit. A cause de ce dernier terme qui dépend du temps via l'intensité, la dérivée de $\phi$ est :

$$
\omega=\omega_{0}-\left(\omega_{0} \mathrm{z} \mathrm{n}_{2} / \mathrm{c}\right) \mathrm{dI} / \mathrm{dt}
$$

Dans la montée de l'impulsion, la fréquence diminue à partir de $\omega_{0}$ (glissement vers l'infrarouge) tandis que dans la décroissance de l'impulsion, elle augmente (glissement vers le bleu). A partir d'une impulsion quasi-monochromatique, on obtient de nombreuses composantes spectrales qui peuvent être un vrai continuum de longueurs d'onde allant de l'infrarouge $(\lambda \geq 1.5 \mu \mathrm{m})$ à l'ultraviolet $(\lambda<0.35 \mu \mathrm{m})$. Il faut remarquer que ce glissement de fréquences a lieu pendant l'impulsion, donc que la durée du continuum n'est pas supérieure à celle de l'impulsion initiale. C'est donc un outil idéal pour faire de la spectroscopie résolue en temps.

Cette même génération de continuum peut aussi être utilisée pour le faisceau de pompe : on sélectionne dans le continuum une longueur d'onde intéressante pour l'excitation et, comme il n'y a pas beaucoup d'énergie dans un intervalle spectral, on amplifie le faisceau jusqu'au niveau d'intensité désiré.

\subsection{Diversité des mesures résolues en temps}

Le dernier schéma donné ci-dessus est caractéristique d'une absorption résolue en temps. Toutefois c'est loin d'être la seule mesure possible et toutes les méthodes d'optique trouvent leur prolongement dans le domaine des temps ultra-courts. Par exemple, la réflectivité résolue en temps s'obtient en recueillant le faisceau test réfléchi et non plus transmis. Même chose pour des mesures de polarisation en introduisant les polariseurs et analyseurs nécessaires. On mesure ainsi des rotations de polarisation ou des anisotropies induites transitoirement par le faisceau excitateur (effet Kerr optique). Deux faisceaux peuvent être envoyés simultanément sur un matériau et y former un réseau par effet non-linéaire. Un troisième faisceau vient lire ce réseau en se diffractant dessus (un des deux faisceaux initiaux peut d'ailleurs être utilisé pour cela). La mesure par diffraction d'électrons est également possible: alors qu'une partie du faisceau laser 
sert toujours à exciter le matériau, une autre partie frappe une photocathode qui émet alors une bouffée d'électrons subpicoseconde. Après accélération, ce paquet d'électrons est diffracté sur le matériau excité par l'impulsion laser et la variation du spectre de diffraction en fonction du délai entre les deux impulsions renseigne sur l'évolution des paramètres cristallins.

A la place du faisceau d'électrons qui se propage dans le vide, on peut également exciter une impulsion électrique sur des lignes conductrices coplanaires où elles vont se propager (cette partie sera développée ultérieurement). Dans un futur extrêmement proche, il est aussi envisagé d'envoyer comme test un faisceau de rayons X généré lui aussi par une partie de l'impulsion laser initiale. Cette énumération peut paraître un peu fastidieuse, mais elle montre la généralité de ces méthodes de mesure temporelle. La luminescence résolue en temps peut sembler entrer dans une autre catégorie : il n'en est rien et nous allons développer un peu cette technique dans la suite.

\subsection{Luminescence résolue en temps}

Les caméras à balayage de fente permettent d'analyser temporellement un signal émis. Bien que des progrès constants augmentent leurs performances, elles n'atteignent pas encore la résolution des méthodes purement optiques et les autres avantages qu'elles procurent sont à mettre en balance avec leur coût.

Les autres méthodes utilisent le principe de l'échantillonnage optique et chacune présente des avantages et des inconvénients. Selon le signal à étudier, il vaudra mieux recourir à l'une ou l'autre.

\subsubsection{La porte optique de effet Kerr}

repose sur l'utilisation d'un obturateur dont l'ouverture est commandée par l'impulsion laser. Une cuve d'un liquide à grand coefficient Kerr est placée entre deux polariseurs croisés $\mathrm{P}_{1}$ et $\mathrm{P}_{2}$.

Le liquide est isotrope en l'absence d'excitation, aussi le signal envoyé est-il bloqué par le deuxième polariseur (partie supérieure de la figure 5). Lorsque une impulsion laser intense est envoyée sur la cuve (partie inférieure de la figure 5), elle induit un indice anisotrope dans le milieu par effet Kerr optique. Si sa polarisation fait un angle avec les deux directions de polarisation définies par $\mathrm{P}_{1}$ et $\mathrm{P}_{2}$, le signal traversant la cuve subit une rotation de sa polarisation et est transmis à travers l'analyseur $\mathrm{P}_{2}$. Lorsque le temps de réponse du milieu est ultra-court (le benzène ou $\mathrm{CS}_{2}$ ont une importante réponse électronique, donc rapide), l'anisotropie ne dure que pendant la durée de l'impulsion laser. En choisissant des cuves minces pour minimiser l'effet des différences de vitesse dans le milieu selon les longueurs d'onde, on obtient une porte optique qui ne s'ouvre que pendant un temps très court.

Les avantages de cette méthode sont sa bonne résolution temporelle et le fait que toutes les composantes spectrales du signal sont transmises ensemble à un délai donné. Il est alors facile d'avoir sur un analyseur multicannal le spectre du signal en fonction du temps. Ses inconvénients sont sa limitation au visible et proche infrarouge et la nécessité de disposer d'impulsions intenses pour induire un effet Kerr suffisant.

\subsubsection{La conversion de fréquence}

utilise l'addition (ou la soustraction) des fréquences de deux faisceaux lumineux dans des matériaux non-centrosymétriques (KDP, BBO, ...). Le signal à analyser (la luminescence par exemple) et une impulsion laser sont envoyés ensemble dans le cristal. En recueillant à la sortie le signal à la fréquence somme des deux fréquences initiales, seule est analysée la partie de la luminescence en coïncidence temporelle avec le laser dans le cristal, c'est à dire une tranche ayant la durée de l'impulsion. 

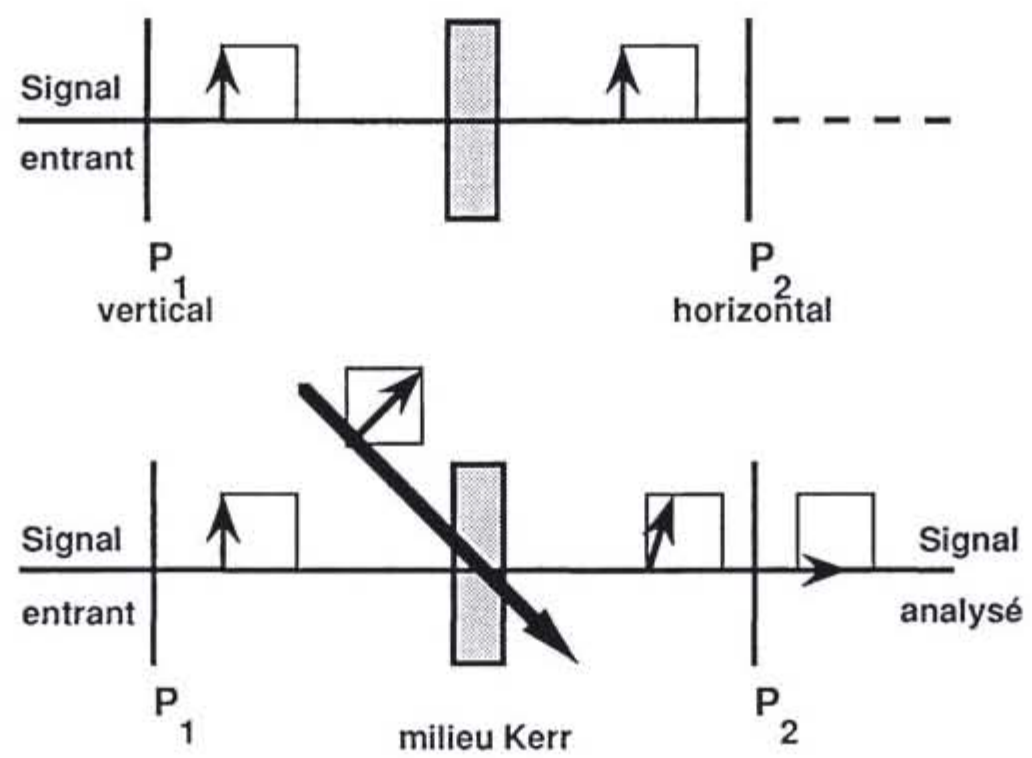

Figure 5 : Principe de l'échantillonnage par effet Kerr. Les petits carrés et les polarisations sont en fait perpendiculaires à la figure.

Les avantages de cette méthode sont sa bonne résolution temporelle (attention toujours aux différences de vitesse selon les longueurs d'onde dans le cristal doubleur) et son utilisation facile pour des signaux infrarouges qui sont transformés en signaux dans le visible. Ses inconvénients sont un faible rendement (compensé par une bonne détectivité dans le visible) et surtout le fait que les règles d'accord de phase imposent un angle différent du cristal pour chaque longueur d'onde.

\subsubsection{L'amplification paramétrique (méthode PASS)}

peut aussi être utilisée pour résoudre temporellement un signal faible (Hulin et al., 1988). Le schéma expérimental est le même que celui de la méthode ci-dessus, mais au lieu d'utiliser la conversion de fréquence, c'est l'amplification paramétrique de la partie du signal en coïncidence temporelle avec l'impulsion laser dans le cristal adéquat qui est utilisée (figure 6). Cette partie ne change pas de fréquence mais voit son intensité multipliée par le facteur de gain ( $10^{4}$ à $\left.10^{8}\right)$. Ce cristal est un cristal non-linéaire comme le BBO ou le NPP (cristal organique).

Le détecteur intégrateur récupère un signal qui est l'intégrale de l'aire sous la courbe de droite (après le cristal). Il mesurera à la longueur d'onde du signal, d'une part une valeur indépendante du délai et qui représente l'intégrale du signal initial, d'autre part une composante dépendante du délai qui est la valeur du signal en cöncidence avec la pompe multipliée par $10^{4}$ à $10^{8}$. En faisant varier le délai entre le début du signal et l'impulsion laser de pompe, toute la courbe du signal va être échantillonnée.

Les avantages de cette méthode sont sa résolution temporelle et sa bonne sensibilité puisque le signal est non pas réduit comme dans les autres méthodes mais amplifié. En travaillant à la longueur d'onde de l'idler, on s'affranchit du fond continu correspondant à l'intégrale du signal non amplifié. Par contre les règles d'accord de phase imposent aussi de travailler longueur d'onde par longueur d'onde, sauf si la chance permet de travailler dans une région spectrale non-critique du cristal. 


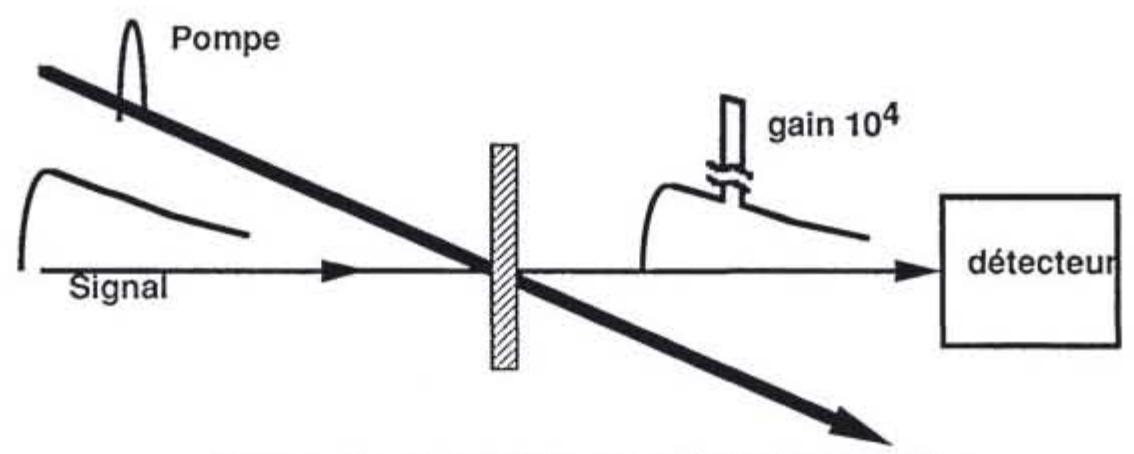

Figure 6 : Principe de résolution temporelle par effet paramétrique

\subsubsection{La conclusion}

qui s'impose à l'examen des trois méthodes ci-dessus, c'est qu'elles reposent elles aussi sur une variante de la méthode pompe-test. Dans les trois cas, une première impulsion génère le signal à étudier et une deuxième vient l'analyser temporellement.

\section{LA PHYSIQUE AUX TEMPS COURTS}

Notre perception d'un phénomène dépend de l'échelle de temps sur laquelle il est regardé. De nombreux processus physiques ("physique" pris au sens le plus général du terme) se passent en des temps très courts, signe que l'interaction correspondante est très forte. Une énumération en serait fastidieuse, aussi la suite de ce chapitre sera plutôt une suite d'exemples servant à illustrer dans différents domaines les apports des études avec des impulsions femtosecondes.

\subsection{Visualisation}

Ainsi que cela a été déjà mentionné, les impulsions lasers femtosecondes peuvent être utilisées pour une visualisation en super-ralenti, un peu comme avec un super-stroboscope. Ainsi que pour la stroboscopie, on tire parti de la répétitivité du phénomène et le retard de l'éclairement est varié entre chaque coup: c'est le principe de la méthode pompe-test.

Cette technique a été utilisée pour visualiser l'aspect de la surface d'un matériau lors de sa fusion après une forte excitation laser (figure 7). Un faisceau laser femtoseconde (durée de l'impulsion : $100 \mathrm{fs}$, longueur d'onde : $620 \mathrm{~nm}$ ) est focalisé sur une plaque de silicium qui est déplacée entre chaque coup pour garder une surface fraîche à chaque nouvelle excitation. L'énergie déposée dans un petit volume est suffisante pour fondre le matériau. Toutefois, la fusion n'est pas immédiate et est précédée par une phase où existe un plasma d'électrons-trous très chaud (quelques milliers de degrés) qui n'est pas en équilibre thermique avec le réseau.

L'échantillon est dans le noir. La région de l'impact de l'excitation intense est éclairée par le test à une autre longueur d'onde que celle de l'excitation et cette image est visualisée sur une caméra ou des réseaux de diodes (Downer et al., 1983). En faisant varier la longueur d'onde du test, on obtient les caractéristiques du plasma transitoire, celles du liquide fondu puis de la solidification. Nous reviendrons sur les toutes premières étapes; aux "longs" temps, il a été montré que cette excitation rapide n'autorise pas une bonne propagation de la chaleur et qu'au refroidissement, il y a amorphisation et non recristallisation. 

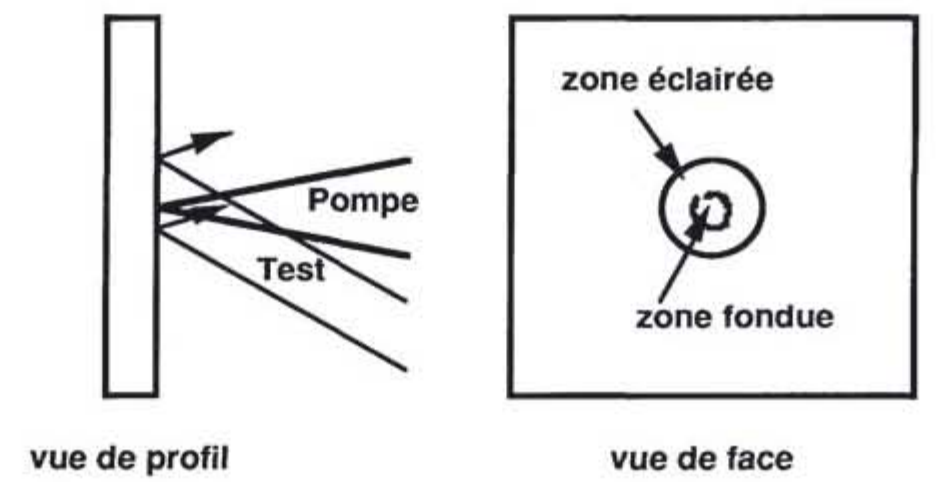

Figure 7: Schéma d'une expérience de visualisation résolue en temps appliquée à la fusion du silicium.

Dans les visualisations de forme, il ne sera mentionné que pour mémoire l'imagerie en milieu diffusant. Il y en existe différentes variantes, mais un très bon exemple a été donné par A. Brun et $\mathrm{P}$. Georges dans ce livre au chapitre des lasers femtosecondes.

\subsection{Mesure des durées de vie}

C'est certainement l'application à laquelle les expérimentateurs ont pensé en premier.

\subsubsection{Durée de vie des porteurs de charge}

Lorsqu'un photon d'énergie suffisante est absorbé par un semiconducteur, il fait passer un électron de la bande de valence vers la bande de conduction; la place vide qu'il laisse dans la bande de valence est appelée trou et se comporte comme un électron de charge positive et d'énergic cinétique négative. Dans un atome ou pour les impuretés dans un isolant, le photon fait passer un électron d'un état de base vers un état excité. Les durées de vie dans ces derniers cas sont assez longues et il n'a pas été besoin d'attendre les lasers femtosecondes pour les mesurer. Par contre, dans les semiconducteurs, les charges sont libres de se déplacer et de rencontrer des centres recombinants.

L'allongement des temps de vie dans un semiconducteur a longtemps traduit l'amélioration de la qualité des matériaux. Pour donner un exemple, on trouve 4 ns dans du bon GaAs massif et quelques centaines de femtosecondes dans la plupart des puits quantiques GaAs/AlGaAS, tous étant des gaps directs (à comparer avec les microsecondes dans les gaps indirects).

Toutefois, il est des cas où une courte durée de vie est spécialement recherchée. Cela arrive dans les expériences où sont générées des impulsions électriques ultracourtes grâce à des lignes métalliques déposées sur un substrat photoconducteur. Le temps de montée de l'impulsion est alors gouverné par la durée de l'impulsion laser et le temps de descente par la durée de vie des porteurs photocréés. Nous reviendrons plus en détail sur ce sujet dans la partie sur l'électrooptique.

La réduction du temps de vie des porteurs libres est obtenue en créant des défauts qui serviront de pièges (réduction de la conduction) ou de centres recombinants. Jusqu'à récemment, cela été obtenu par bombardement avec des protons. Cette méthode donne des résultats intéressants, mais la dose d'irradiation est limitée car si elle est trop importante, le grand nombre de défauts créés réduit fortement la mobilité; cela peut même aller jusqu'à l'amorphisation du cristal.

Depuis peu, les lignes métalliques sont déposées sur des couches de GaAs élaborées à basse température. Au lieu des $\approx 700^{\circ} \mathrm{C}$ habituels du substrat pour épitaxier une bonne couche 
cristalline, la température est descendue à $=180^{\circ} \mathrm{C}$ sous excès d'arsenic. Des études microscopiques ont montré qu'il se forme alors dans la couche déposée des micro-agrégats d'arsenic qui servent de pièges efficaces pour les porteurs, sans toutefois réduire la mobilité dans le reste du matériau. La durée de vie est réduite à une fraction de picoseconde.

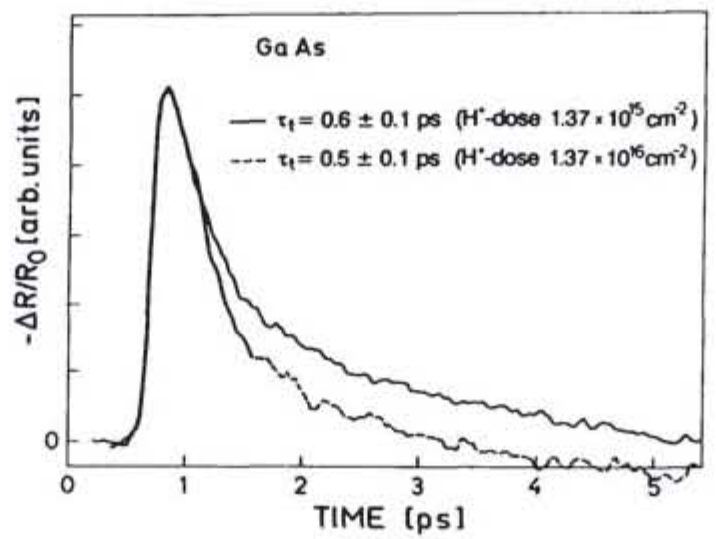

Figure 8 : Mesure de la durée de vie des porteurs dans un échantillon de GaAs bombardé par des protons. La courbe en traits fins ( $\mathrm{t}=0.6 \pm 0.1 \mathrm{ps}$ ) a été obtenue pour une dose de protons de $10^{15} \mathrm{~cm}^{-2}$ et la courbe en trait gras ( $t=0.5 \pm 0.1 \mathrm{ps}$ ) pour une dose de $10^{16} \mathrm{~cm}^{-2}$. La mesure est faite en mesurant la durée du changement de réflectivité après excitation optique de porteurs libres. D'après Lambsdorff et al. 1991.

\subsubsection{Durée de vie des phonons}

Les électrons ne sont pas les seules particules dont on souhaite mesurer la durée de vie. En particulier les phonons, qui sont les vibrations quantifiées du réseau, jouent un rôle fondamental dans l'interaction entre le système électronique et les atomes.

Les phonons peuvent être mesurés par la lumière grâce en particulier à l'effet Raman.

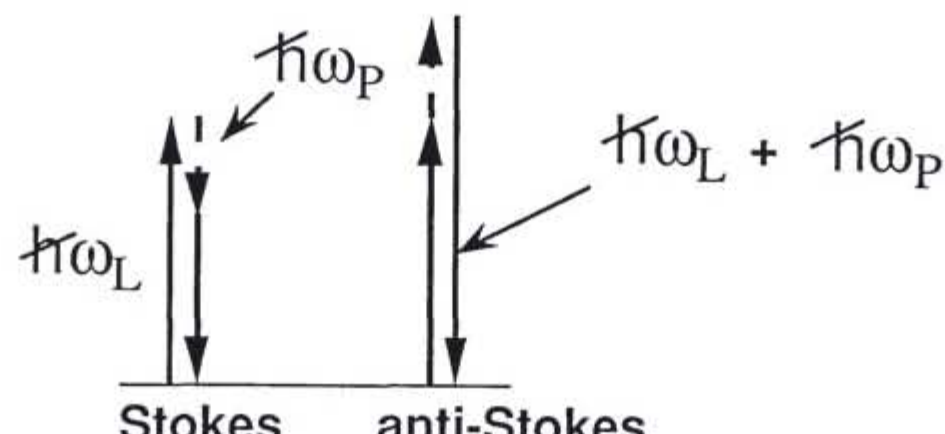

Figure 9 : Effet Raman où un photon envoyé dans le matériau à la fréquence $\omega$ L ressort après avoir émis un phonon à la fréquence $\omega \mathrm{P}$ (effet Stokes) ou absorbé un phonon (effet anti-Stokes) déjà présent dans le matériau. Le niveau excité peut être virtuel ou réel; dans ce dernier cas, on parle d'effet Raman résonnant. 
L'écart de la raie Raman à la raie laser mesure l'énergie du phonon. La raie anti-Stokes n'existe qu'en présence de phonons. La mesure de l'intensité de cette raie est donc un moyen de mesurer leur population dans un matériau.

Dans l'expérience ci-dessous (J.F. Young, 1994), des phonons optiques sont créés lors de la thermalisation de porteurs dans GaAs excités par une impulsion femtoseconde. Une seconde impulsion laser induit un effet Raman et permet la mesure l'intensité de la raie anti-Stokes en fonction du temps. Young a ainsi pu montrer que la durée de vie des phonons optiques, habituellement de 8 ps dans GaAs (von der Linde, 1980), est réduite en présence d'un plasma d'électrons-trous, jusqu'à une valeur de 2 ps pour une densité de $1.510^{11} \mathrm{~cm}^{-2}$.

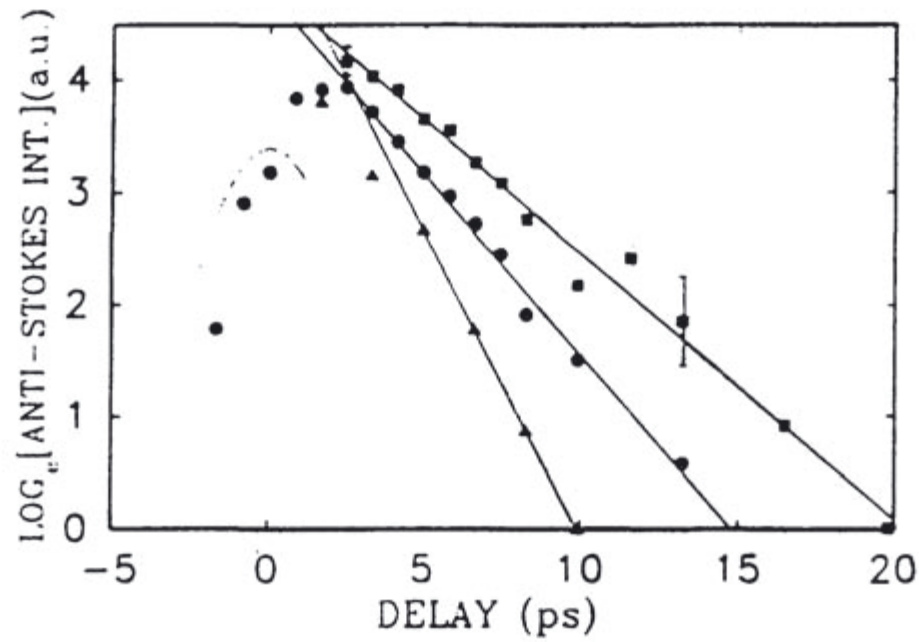

Figure 10 : Intensité de la raie anti-Stokes en fonction du délai entre les impulsions de pompe et de test. Les trois types de données correspondent à des densités de plasma de $0,1.5$ et $3 \cdot 10^{11} \mathrm{~cm}^{-2}$. D'après J.F. Young, 1994, droits réservés.

\subsection{Durée de vie dans un état excité}

Les deux paragraphes ci-dessus se rapportent déjà à des durées de vie après excitation, mais c'était l'état excité dans sa globalité qui était considéré par rapport à l'état de base. Maintenant, nous nous intéressons au temps que reste un porteur dans un état excité déterminé. C'est un sujet très vaste, mais nous examinerons principalement le problème du temps de capture dans un puits quantique.

\subsubsection{Capture par un puits quantique}

La bande d'énergie interdite (le gap) dans un semiconducteur dépend essentiellement de la nature de celui-ci. En incluant une couche mince d'un semiconducteur de faible gap entre deux autres de plus grand gap, on fabrique un puits de potentiel où les particules excitées dans 
l'ensemble du système vont venir se rassembler après un certain temps. La mesure de ce temps est importante pour les dispositifs comme les diodes lasers ou certains transistors.
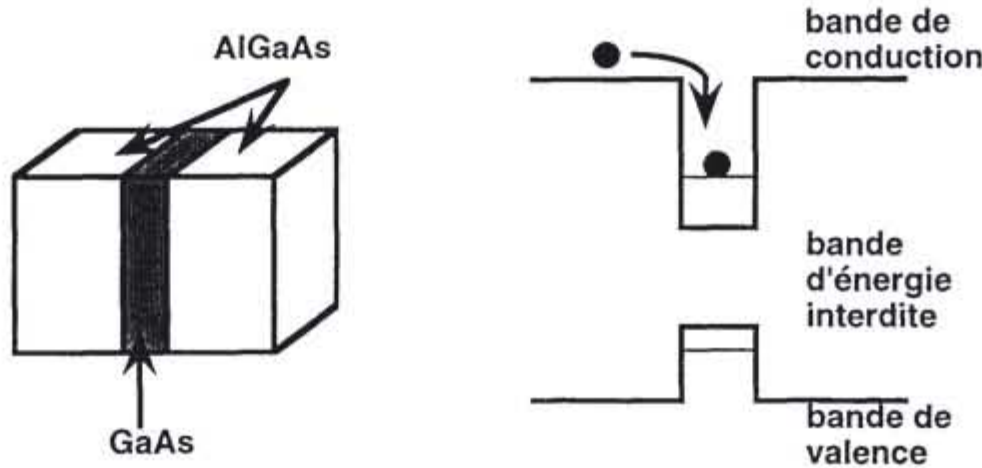

Figure 11 : Représentation schématique d'un puits quantique de GaAs entre deux couches d'AlGaAs de plus large bande d'énergie interdite: à gauche, le matériau, et à droite, la structure de bande. Le dessin de droite figure schématiquement la capture dans le puits d'un électron excité dans l'AlGaAs.

La physique qui gouverne ce processus n'est pas simple car lorsque le puits de potentiel est étroit, il s'y produit une quantification des niveaux d'énergie (puits quantique) (pour une revue, voir Bastard G. et Brum J.A., 1986). Certains de ces niveaux sont dans le puits, d'autres au dessus, et il reste encore des controverses sur l'interprétation des résultats expérimentaux. Dans certaines conditions, la théorie prédit des temps assez longs (Brum J.A. et Bastard G., 1986) alors que les expériences trouveraient plutôt un temps rapide (de l'ordre de la picoseconde ou moins) (Deveaud B. et al., 1988).

\subsubsection{Effet tunnel}

Il peut y avoir plusieurs puits quantiques dans une structure. Si l'épaisseur qui les sépare est grande (supérieure à $8 \mathrm{~nm}$ ), la barrière entre eux est dite épaisse et les puits sont isolés les uns des autres. Si la barrière est mince, il existe un couplage entre les puits par effet tunnel à travers la barrière.
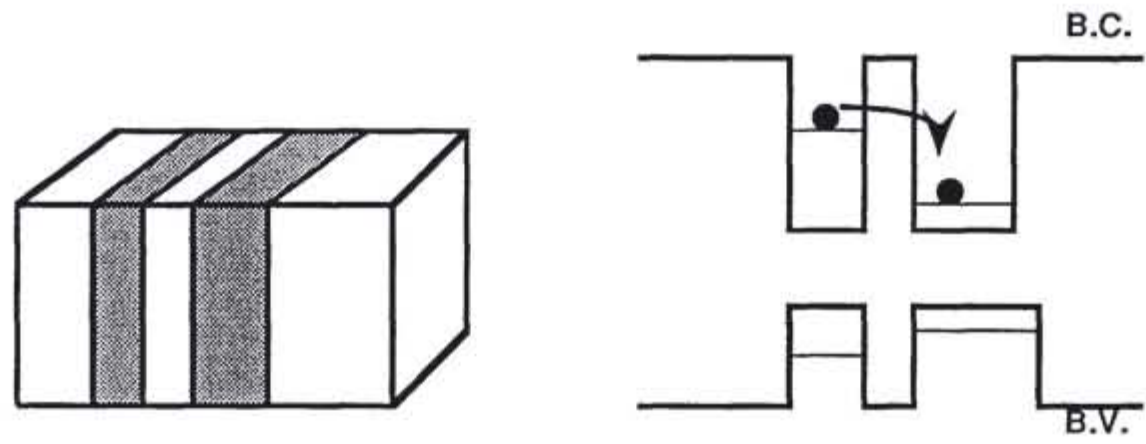

Figure 12 : Représentation schématique de deux puits quantiques de largeurs différentes couplés par effet tunnel à travers une barrière mince. 


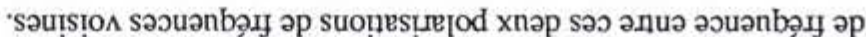

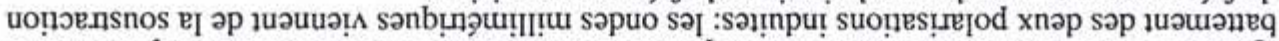

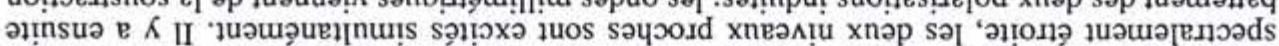

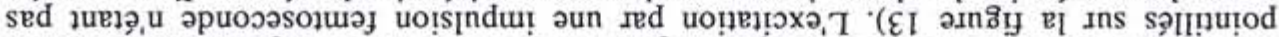

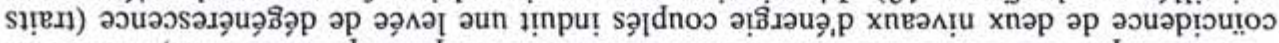

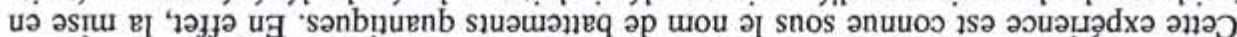

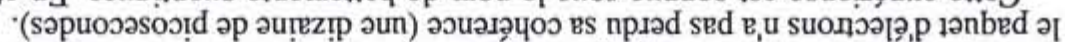

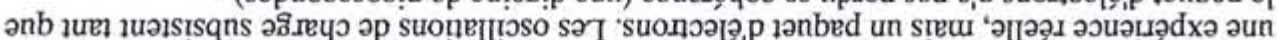

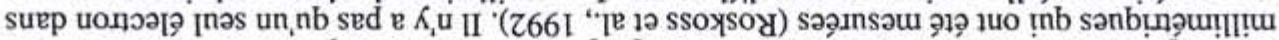

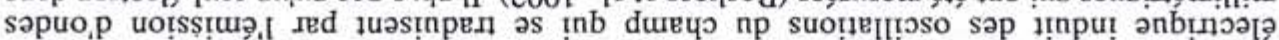

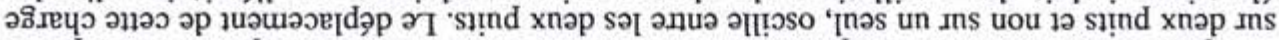

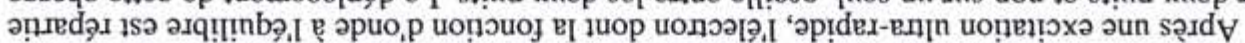

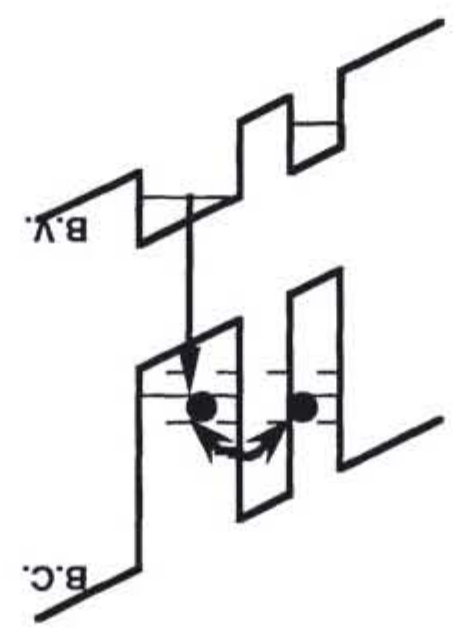

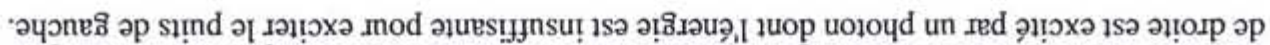
sı!nd ә ן

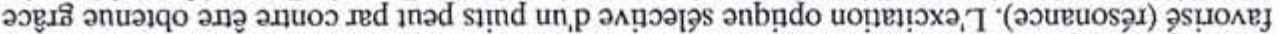

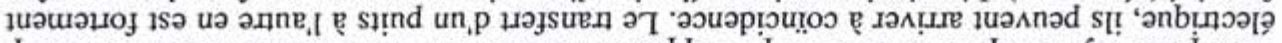

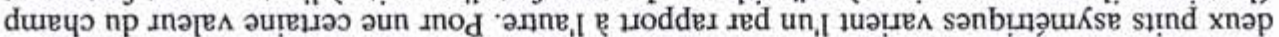

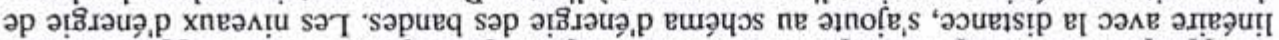

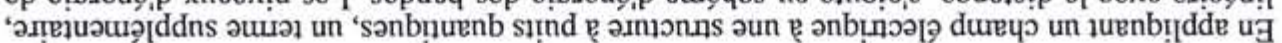

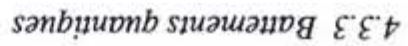

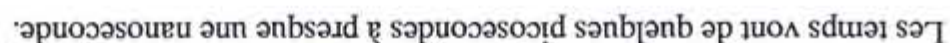

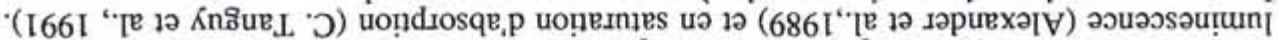

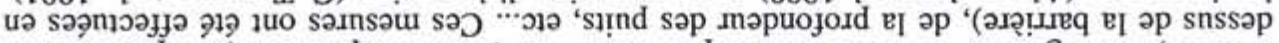

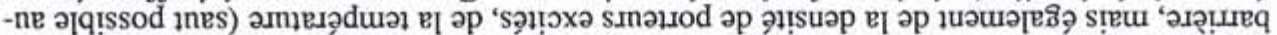
६

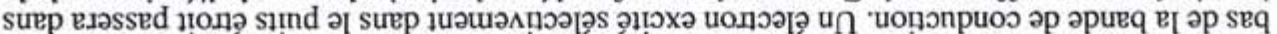

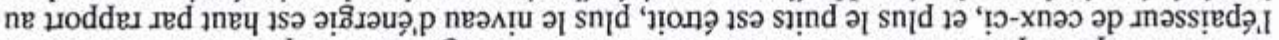

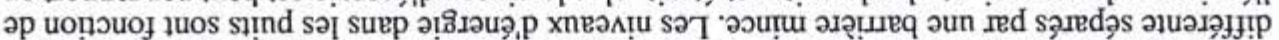

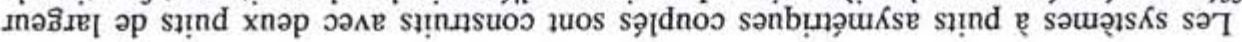




\subsection{Relaxation de l'énergie dans un amorphe}

Le silicium amorphe présente l'avantage sur le silicium cristallin de pouvoir être préparé en grande surface à moindre coût. En particulier, il est utilisé pour les grands écrans de détection. Ceci est particulièrement utile dans les domaines spectraux où la réduction d'image par focalisation est quasi impossible comme les rayons $\mathrm{X}$.

Cependant la sensibilité de détection de l'amorphe est moyenne car les porteurs libres ont une mobilité faible et une durée de vie courte. Pour compenser cela, il faudrait pouvoir utiliser l'amplification produite dans les photodiodes à avalanche. Rappelons brièvement l'effet d'avalanche: une charge est accélérée par un fort champ électrique (en général dans la zone de transition d'une diode). Sous cette action, elle gagne de l'énergie et si elle acquiert une énergie supérieure à l'énergie de la bande interdite, elle peut générer par collision sur le système une paire électron-trou en perdant l'énergie correspondante. Ces deux charges ont été créées dans la zone du champ électrique et à leur tour sont accélérées jusqu'à ce que chacune génère un électron et un trou qui à leur tour,...Une charge est donc ainsi fortement multipliée.

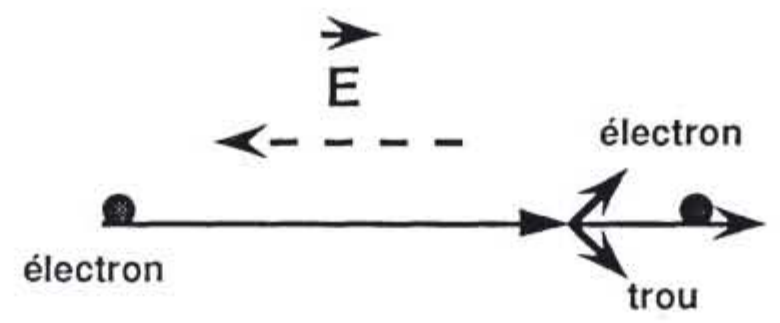

Figure 14 : Accélération d'un électron par un fort champ électrique et production d'une paire électron-trou supplémentaire.

Quels que soient les dispositifs construits et les champs appliqués, un tel effet d'avalanche n'a pas encore été observé dans le silicium amorphe. En fait, il y a dans tout matériau, compétition entre le gain d'énergie par l'action du champ électrique et la perte d'énergie par relaxation vers le réseau. Ce dernier paramètre est mal connu dans les amorphes car les mesures de spectroscopie y sont difficiles.

Pour mesurer le temps nécessaire à la relaxation de l'énergie dans le silicium amorphe, l'expérience suivante a été faite avec des lasers femtosecondes (Mourchid et al., 1990). Une première impulsion dans le visible crée une population d'électrons et de trous sans excès d'énergie. Une deuxième impulsion vient chauffer ces porteurs sans en changer le nombre. Pour cela, sa longueur d'onde est choisie dans l'infrarouge où se produit une forte absorption par porteur libre (le photon donne son énergie à un électron ou à un trou), mais plus d'absorption bande à bande.

Il suffit de mesurer le temps d'échauffement du réseau après l'excitation par le laser IR, ceci avec un faisceau test qui mesure la réduction de la bande d'énergie interdite avec la température. Les expériences ont montré que ce temps est malheureusement très court (une centaine de femtosecondes), ce qui explique pourquoi l'effet d'avalanche est difficile à observer dans le silicium amorphe.

Ce résultat est à rapprocher d'autres mesures dans les amorphes qui donnent souvent des temps plus courts que pour le cristal correspondant. Ceci est probablement dû au fait que l'interaction avec un matériau amorphe reste soumise à la loi de conservation de l'énergie mais que la conservation du moment est moins bien définie. La levée de cette dernière restriction sur les états finaux rend les processus plus probables, donc plus rapides. 


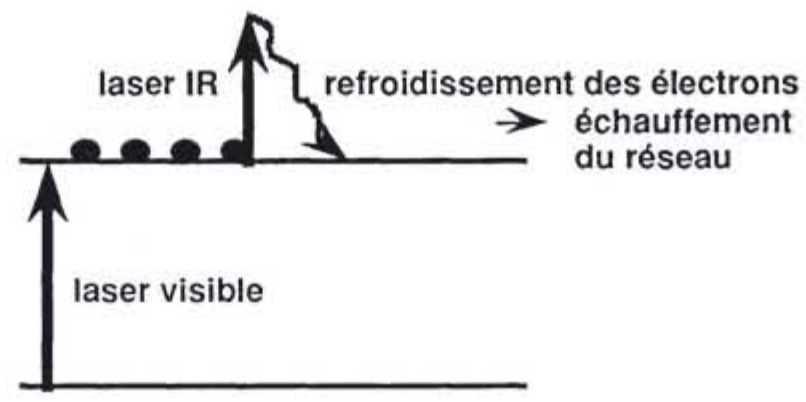

Figure 15 : Principe de l'expérience femtoseconde utilisée pour mesurer le temps de relaxation de l'énergie des électrons dans le silicium amorphe.

\subsection{Situations hors d'équilibre}

L'étude des états excités est déjà en soi une étude de systèmes qui ne sont pas à l'équilibre thermodynamique complet. Cependant il existe des gradations dans les systèmes hors d'équilibre et ce terme est en général réservé à des situations qui durent extrêmement peu et qui ne sont donc observables que grâce aux expériences faites avec des lasers femtosecondes.

\subsection{1 Électrons hors d'équilibre dans GaAs}

Un exemple parlant est donné par l'excitation de porteurs dans de l'arséniure de gallium massif. Un photon d'énergie supérieure à la bande d'énergie interdite peut être absorbé dans le semiconducteur (transition bande à bande) et créer des électrons et des trous à une position bien déterminée dans les bandes à cause de la loi de conservation de l'énergie et du moment.

Cette situation juste après l'excitation est tout à fait loin de l'état d'équilibre. Dans un premier temps, si la densité de porteurs est suffisante, il y a d'abord interaction entre les porteurs (collisions) et l'établissement d'une distribution thermale pour les porteurs. On appelle distribution thermique ou thermale , une répartition en énergie qui suit la distribution de Fermi (de Bose pour les bosons) avec un paramètre variable qui est la température. Cette température est d'abord différente de celle du réseau. Par échange d'énergie entre le système électronique et le réseau via l'émission de phonons, la température électronique va progressivement tendre vers celle du réseau. Ensuite, sur des temps beaucoup plus longs, il y aura recombinaison des électrons avec les trous pour atteindre l'équilibre complet.

Il y a donc des étapes (presque) distinctes dans la thermalisation des porteurs de charge, étapes qui sont toutes hors de l'équilibre, mais il est certain que c'est la première distribution, avant toute thermalisation des électrons, qui est le plus hors d'équilibre.

Il est possible de suivre l'évolution de la distribution des porteurs grâce aux expériences pompe et test avec des lasers femtosecondes. Les électrons étant des fermions, les positions qu'ils occupent dans l'espace des moments ne peuvent être occupées une deuxième fois. Il y a donc une diminution de l'absorption à l'énergie correspondante, diminution proportionnelle au nombre d'électrons à cette énergie. En mesurant l'absorption différentielle (précisément $-\Delta \alpha$ ) en fonction de l'énergie pour un délai connu après l'excitation, on a directement l'évolution du profil de la distribution des électrons. Ce type d'expérience est appelé "hole-burning" ou saturation sélective d'états. 


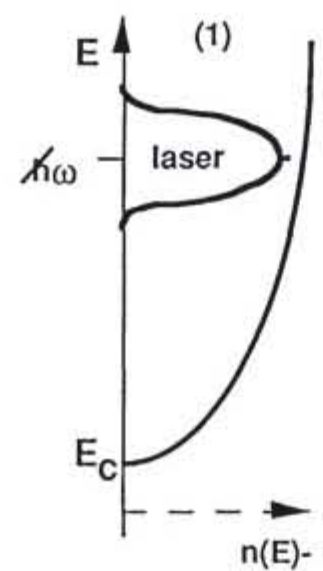

distribution

non

thermalisée

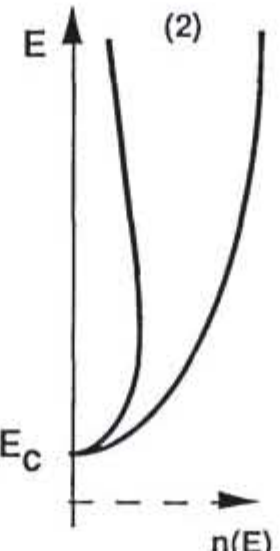

$$
\begin{gathered}
\text { distribution } \\
\text { thermalisée } \\
\text { chaude }
\end{gathered}
$$

(3)

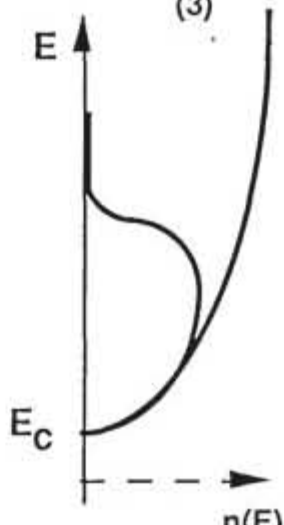

$n(E)$

Figure 16 : Juste après l'cxcitation (cas 1), la distribution des électrons est athermale, c'est-à-dire qu'elle ne peut pas être décrite par une distribution thermalisée, quelle qu'en soit la température. La largeur de la distribution reflète la largeur spectrale du laser. Dans le cas (2), les électrons sont thermalisés entre eux, mais pas encore avec le réseau. La température électronique est élevée car elle résulte d'une répartition de l'énergie initiale entre les électrons. Dans le cas (3), la thermalisation avec le réseau a lieu et la température est celle du réseau. Les aires sous les trois courbes sont égales dans l'hypothèse où il n'y a pas encore eu de recombinaison de porteurs.

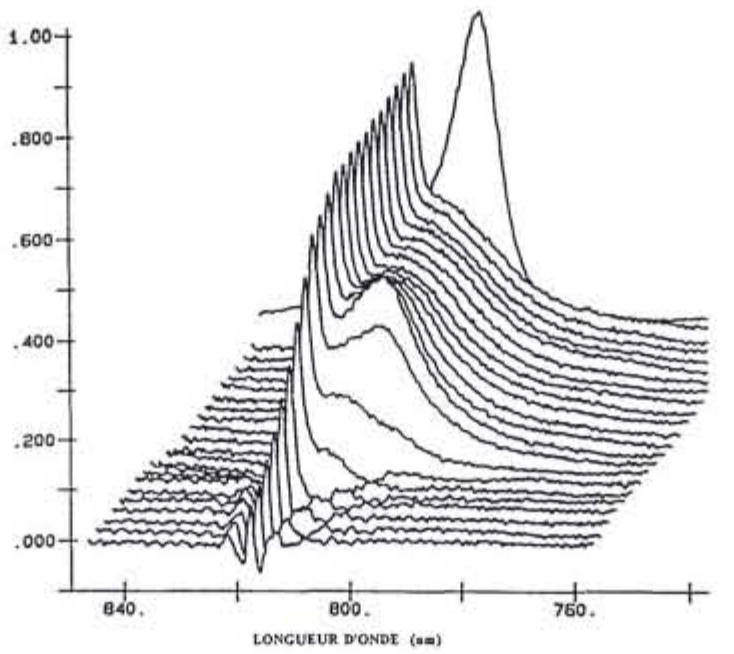

Figure 17: Transmittance (= -absorption) différentielle résolue en temps de GaAs à $15 \mathrm{~K}$ lors d'une excitation à $807 \mathrm{~nm}$. La dernière courbe représente le profil de la pompe. Le test a une durée inférieure à $100 \mathrm{fs}$, mais la pompe est un peu plus longue à cause de son étroitesse spectrale. Les 11 premières courbes sont enregistréses avec un écart entre elles de 60 fs, les 9 suivantes avec un écart de 100 fs et les deux dernières avec un écart de $1 \mathrm{ps}$. La demière courbe correspond donc à un retard de 3 ps après le maximum de l'excitation. La raie observée autour de $819 \mathrm{~nm}$ correspond à la disparition de l'absorption excitonique. Ce qui apparât à plus courte longueur d'onde donne la distribution des porteurs. 
Pour faire comprendre le principe de ces expériences, la description en a été volontairement très simplifiée. D'abord la mesure d'un élargissement de la distribution initiale par rapport à la largeur du laser donne la valeur du temps de déphasage $T_{2}$ (Oudar et al., 1985). Ensuite, il est connu que des fermions à forte densité ont des interactions particulières. La situation a été étudiée à l'équilibre lorsque les électrons sont rassemblés dans une mer de Fermi. La distribution hors d'équilibre a permis d'étudier ce qui se passait dans un cas plus général et de mettre en évidence des effets nouveaux (J.P. Foing et al., 1992). Le retour à l'équilibre donne des indications sur les interactions électron-électron ou électron-phonon, ceci pour différentes densités de porteurs.

\subsubsection{La fusion froide dans le silicium}

Lorsqu'une impulsion lumineuse est absorbée par un semiconducteur dans une transition bande à bande, elle fait passer des électrons de la bande de valence vers la bande de conduction. Dans un solide covalent comme le silicium, il est presque équivalent de dire que les électrons sont passées d'orbitales de valence liantes vers des orbitales excitées anti-liantes. Si le nombre d'électrons excités est proche du nombre total des électrons qui forment les liaisons de valence la cohésion du système n'est plus maintenue, le cristal rigide cesse d'exister et il fond. Ce qui est tout à fait extraordinaire dans ce mécanisme, c'est qu'il se produit sans aucun apport de chaleur au réseau qui reste froid, d'où le terme de fusion froide.

Cette fusion froide est à comparer au mécanisme habituel de fusion par apport de chaleur. L'agitation des atomes de silicium autour de leur position d'équilibre augmente lorsque la température augmente, et si l'écart moyen dépasse une fraction d'environ un dixième de la distance interatomique, il y a rupture de la liaison rigide et le cristal fond.

Toutefois les deux situations ne sont différentes que pendant un court intervalle de temps. En effet, dans la fusion froide, il y a bien eu dépôt d'énergie mais vers le système électronique. Celui-ci est en interaction avec le réseau par échange de phonons et rapidement, il cède son excès d'énergie aux ions de silicium. La température de ceux-ci augmente et bientôt dépasse la température de fusion normale. Les situations sont alors équivalentes.

Des évaluations théoriques ont estimé que le temps pendant lequel existait un liquide froid était de l'ordre de la centaine de femtosecondes. Cette durée est maintenant tout à fait accessible expérimentalement. Le problème reste de faire la mesure qui prouvera vraiment que le liquide froid existe. La lumière visible n'interagit qu'avec le système électronique et celui-ci est par essence complètement hors d'équilibre avec le réseau. Il faudrait avoir recours à de la diffraction d'électrons ou aux rayons X. Les progrès expérimentaux sont tels que cela sera bientôt possible. Il reste un dernier problème, celui de différencier un solide et un liquide froid originaire d'un solide ordonné et qui a peu la possibilité de bouger en $100 \mathrm{fs}$. Peut-être faudraitil prouver la disparition de la composante de cisaillement dans les vibrations du liquide. Le problème reste ouvert, mais c'est un joli exemple de système complètement hors d'équilibre qui ne peut être observé qu'avec des impulsions femtosecondes, une sorte de nouvel état de la matière.

\section{MODULATION ET COMMUTATION OPTIQUE}

\subsection{Introduction}

Le transport et le traitement de l'information peuvent se faire de différentes façons, la plus connue étant électrique. Les possibilités offertes par ces systèmes nous étonnent tous les jours, mais certaines limitations intrinsèques sont prédites. Les moyens optiques sont une alternative intéressante avec des limitations théoriques beaucoup plus reculées que pour les systèmes électroniques. Bien que les développements technologiques actuels ne soient pas très avancés dans ce dernier domaine, il est important d'explorer les possibilités de l'optique en général et des impulsions ultra-courtes en particulier. 

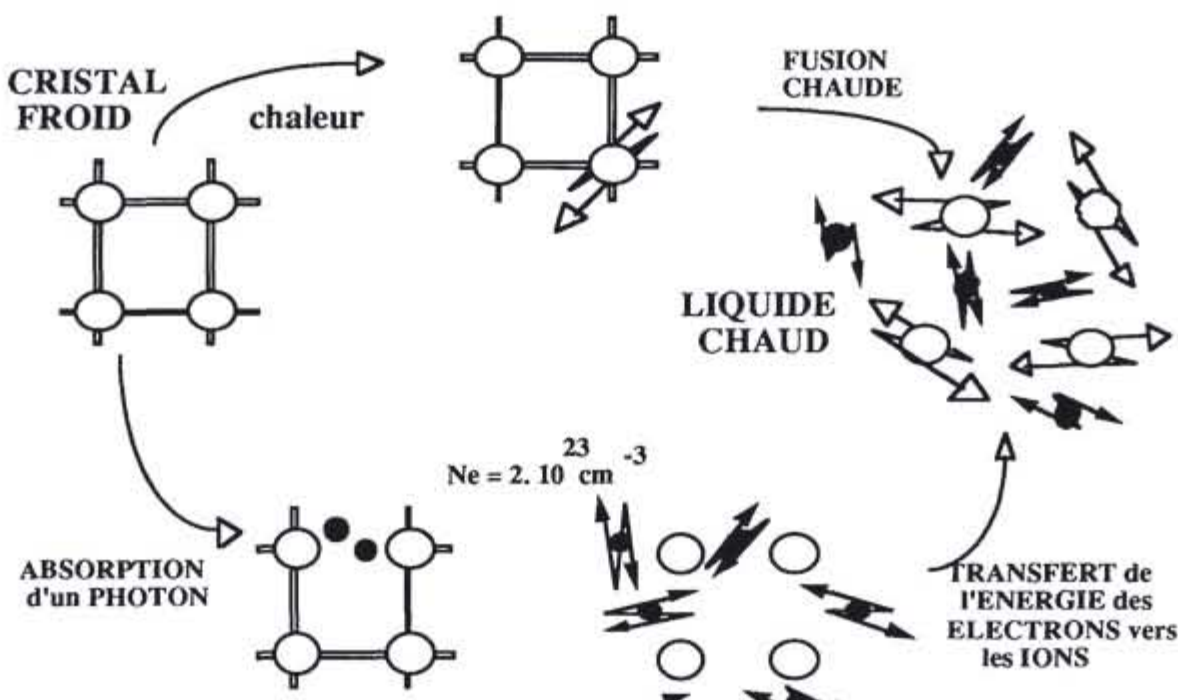

$10 \mathrm{~cm}^{-3}$
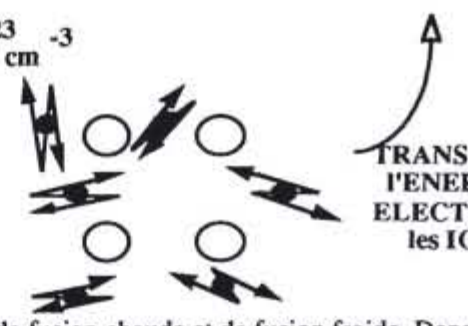

TRANSFERT de I'ENERGIE des les IONS

Figure 18 : Représentation schématique des processus de fusion chaude et de fusion froide. Dans les deux cas, on part du cristal froid non-excité. La fusion classique par chaleur induit un mouvement des atomes et le résultat est le liquide chaud (chemin du haut). Dans la fusion aidée par laser (chemin du bas), un photon absorbé casse la liaison entre deux siliciums sans chauffer les ions. Si un nombre d'électrons proche d'un nombre total est excité, les liaisons n'existent plus mais les ions sont froids (liquide froid). Le transfert d'énergie ultérieur des électrons vers les ions redonne un liquide chaud (étape finale)

Le codage de l'information sous la forme d'impulsions lumineuses commence à être utilisé, en particulier dans les systèmes de transmission à fibres optiques. Pour le traitement de l'information, les méthodes optiques se proposent de dépasser les capacités de l'électronique dans deux domaines. Le premier est le parallélisme, c'est-à-dire la réalisation simultanée d'un grand nombre d'opérations élémentaires. Comme il est difficile de connecter entre eux une myriade de composants électroniques, les performances obtenues dans cette voie par les méthodes actuelles sont assez limitées. La lumière pouvant se propager en l'absence de support matériel, on peut imaginer une collection d'unités élémentaires réalisant en parallèle des opérations très simples et se transmettant l'une à l'autre les résultats par des faisceaux lumineux.

Le deuxième domaine dans lequel l'optique peut dépasser l'électronique est celui de la rapidité . La commutation d'un composant électronique nécessite le déplacement de charges électriques, ce qui limite la vitesse maximum d'opération. De plus, la propagation de la lumière se fait à la plus grande vitesse existante. L'idée d'utiliser des impulsions lumineuses très courtes comme vecteurs de l'information paraît donc séduisante pour augmenter la rapidité d'exécution du traitement de l'information.

Il est donc important de définir des composants optiques qui puissent répondre aux deux critères de parallélisme et de rapidité. Il faut de plus que l'énergie dépensée pour chaque opération soit petite, d'autant plus faible que le nombre de commutation par unité de temps ou de surface est grand. Les impulsions lumineuses ayant traversé un premier dispositif doivent être capables d'en contrôler un autre (cascadabilité) : il faut donc que, à l'exception de la variable modifiée par l'interaction dans le dispositif, leurs caractéristiques physiques ne soient pas changées par la traversée du dispositif. Enfin la technologie proposée doit être économiquement viable, ce qui signifie que l'augmentation des performances apportées par l'optique doit compenser le coût très probablement élevé de l'utilisation de telles techniques. 


\subsection{Remarques sur les dispositifs optiques}

De nombreux dispositifs ont été proposés, satisfaisant un ou plus rarement plusieurs des critères ci-dessus. De grandes familles de composants optiques peuvent être distinguées. Tout d'abord, les composants hybrides permettent de moduler la lumière grâce à l'application d'un champ électrique externe de commande. Ils présentent l'inconvénient d'avoir un temps de réponse qui dépend de l'électronique de commande. La deuxième famille peut se regrouper sous le terme de bistables optiques planaires, avec pour archétype le Fabry-Perot non-linéaire. Enfin une dernière famille de composants de logique optique est apparue récemment et propose des dispositifs de commutation dans les guides d'onde et les fibres optiques. Les plus avancés d'entre eux sont prévus pour fonctionner avec des impulsions de type soliton. La géométrie en guide d'onde rend difficilement imaginable un traitement parallèle des informations. Cette dernière classe de dispositif ne sera pas abordée ici car elle s'apparente au chapitre sur les fibres optiques.

Un faisceau lumineux n'interagissant pas avec un autre faisceau lumineux dans le vide, toute modulation ou commutation de la lumière passe par l'interaction lumière-matière. Cette dernière est décrite dans le formalisme général de la susceptibilité, mais cela regroupe une grande quantité de phénomènes différents. Pour simplifier, on peut dire que tout changement de l'indice de réfraction ou de l'absorption peut permettre de moduler le faisceau lumineux. On peut aussi remarquer que, de façon plus générale, tout changement d'indice peut être relié à un changement d'absorption via les relations de Kramers-Krönig et vice versa. Une fois dites ces grandes généralités, il apparaît que certains dispositifs tirent surtout parti du changement d'indice, d'autres du changement d'absorption à la longueur d'onde du faisceau lumineux.

Parmi les effets physiques capables de faire varier l'indice d'un matériau, plusieurs sont consécutifs à l'éclairement. Les temps de réponse associés dépendent de la nature du processus:

- les effets thermiques proviennent de ce que la lumière absorbée se retrouve à la fin sous forme de chaleur. Les propagations de la chaleur étant des processus assez lents, les temps associés sont de l'ordre de la milliseconde ;

- la polarisation des cristaux liquides se produit sur l'échelle de temps des millisecondes car elle nécessite l'orientation dans l'espace de longues molécules à grand encombrement stérique. En général, la commande en est plutôt électrique, mais cette dernière peut être déclenchée par une commande optique ;

- les effets électroniques dans les semiconducteurs (silicium, arséniure de gallium,...) sont souvent consécutifs à la création d'un plasma de particules chargées. La lumière excite les électrons hors des liaisons de valence où ils sont confinés (bande de valence) pour les amener à un état d'électrons quasi-libres (bande de conduction). Ce plasma de particules quasi-libres influence toutes les propriétés électroniques du matériau et par conséquent les propriétés optiques. Son effet dure pendant le temps de vie des électrons excités, avant qu'ils ne retombent dans la bande de valence. Selon les matériaux, la température ou les défauts introduits, cela va de quelques nanosecondes $\left(10^{-9} \mathrm{~s}\right)$ à quelques picosecondes $\left(10^{-12} \mathrm{~s}\right)$;

- les effets virtuels correspondent à un couplage de la lumière avec les résonances du matériau sans absorption réelle de lumière (effet Kerr, effet Stark optique, ...). Ils ne durent que pendant la durée de l'impulsion lumineuse. Ils peuvent donc être aussi brefs que souhaités en utilisant des impulsions ultra-courtes, mais en contrepartie exigent des puissances plus élevées.

Il ressort de cette présentation générale que le nombre de dispositifs capables de moduler la lumière et de servir dans les commutations optiques peut être très grand. Dans la suite de ce chapitre, la présentation sera limitée à l'étalon de Fabry-Perot non-linéaire qui peut servir de porte optique à commande optique ou de mémoire optique, ceci avec des rapidités de fonctionnement inégalées. 


\subsection{L'étalon de Fabry-Perot non-linéaire}

\subsubsection{Rappels sur le Fabry-Perot}

L'interféromètre de Fabry-Perot (appelé plus simplement par la suite Fabry-Perot) est constitué de deux miroirs plans et parallèles séparés par une distance $\mathbf{a}$. Entre ces deux miroirs est placé un milieu matériel d'indice de réfraction $\mathbf{n}$ tandis qu'à l'extérieur des miroirs, l'indice est supposé égal à 1. Lorsqu'une onde électromagnétique est envoyée sur un Fabry-Perot, elle subit des réflexions multiples entre les deux miroirs. Les ondes peuvent interférer constructivement ou destructivement suivant leurs déphasages relatifs, déphasages qui dépendent du rapport de l'épaisseur optique du Fabry-Perot à la longueur d'onde de la lumière envoyée.

On appelle $\phi$ le déphasage accumulé sur une traversée du Fabry-Perot :

$$
\phi=\frac{\pi}{\lambda} \text { a n }
$$

Dans le cas idéal d'un Fabry-Perot sans absorption avec deux miroirs identiques sans pertes et sans déphasages, la transmission est donnée par la fonction d'Airy :

$$
\mathrm{T}=\left[1+\frac{4 \mathrm{R}}{[1-\mathrm{R}]^{2} \sin ^{2} \phi}\right]
$$

La courbe de transmission présente un maximum chaque fois que le dénominateur devient résonnant, c'est à dire que la relation suivante est vérifiée :

$$
2 \mathrm{na}=\mathrm{p} \lambda \quad \text { avec } \mathrm{p} \text { entier }
$$

Cette condition correspond à un chemin optique pour un aller et retour dans le Fabry-Perot égal à un nombre entier de longueurs d'onde, ce qui assure bien que toutes les ondes s'ajoutent en phase. Les interférences sont parfaitement constructives pour l'onde transmise et destructives pour l'onde réfléchie par le Fabry-Perot ; la transmission est alors égale à l'unité.

\subsubsection{Non-linéarité dispersive}

Si l'indice du matériau n ne peut plus être considéré comme une constante mais dépend de l'intensité lumineuse, on peut développer l'indice en puissance de l'intensité dans le milieu. A l'ordre le plus bas, l'indice de réfraction s'écrit :

$$
\mathrm{n}=\mathrm{n}_{0}+\mathrm{n}_{2} \mathrm{I}
$$

Une hypothèse très importante se cache derrière la relation ci-dessus. La variation de l'indice de téfraction est due à une interaction entre l'onde lumineuse et le milieu qu'elle traverse. Cette interaction est en général un processus complexe et ses effets peuvent se produire avec un certain retard ou avoir une dépendance temporelle notable. La relation ci-dessus ne mentionne pas ce retard, elle dissimule donc l'hypothèse d'un temps de réaction du milieu négligeable devant le temps caractéristique des variations de l'intensité dans le milieu.

\subsubsection{Fabry-Perot non-linéaire}

Si l'indice de réfraction du milieu varie en fonction de l'intensité lumineuse, la longueur d'onde de résonance est modifiée. Le pic de transmission se déplace spectralement. Si le faisceau optique qui traverse le dispositif a une longueur d'onde coïncidant avec la résonance avant perturbation, il se trouve désaccordé et n'est plus transmis mais réfléchi. Si au contraire il était initialement hors résonance, il peut maintenant être transmis. On retrouve le principe d'une porte optique qu'on fait commuter d'un état passant vers un état bloquant (faiblement transmettant) ou bien d'un état bloquant vers un état passant. Comme c'est la lumière qui provoque les variations d'indice, il s'agit d'une porte optique à commande optique. 
L'intensité lumineuse qui sert à modifier l'indice peut venir d'un faisceau différent de celui qui doit être modulé. Le faisceau extérieur commande l'ouverture ou la fermeture de la porte (figure 19). Mais il existe aussi des cas où c'est le faisceau que l'on cherche à moduler qui modifie lui-même l'indice. C'est dans cette dernière situation que l'on peut trouver de la bistabilité optique, c'est à dire deux états possibles de la transmission pour une même valeur de l'intensité incidente. L'état de transmission faible ou forte dépend de l'histoire antérieure de l'éclairement, ce qui peut servir de principe de base aux mémoires optiques.

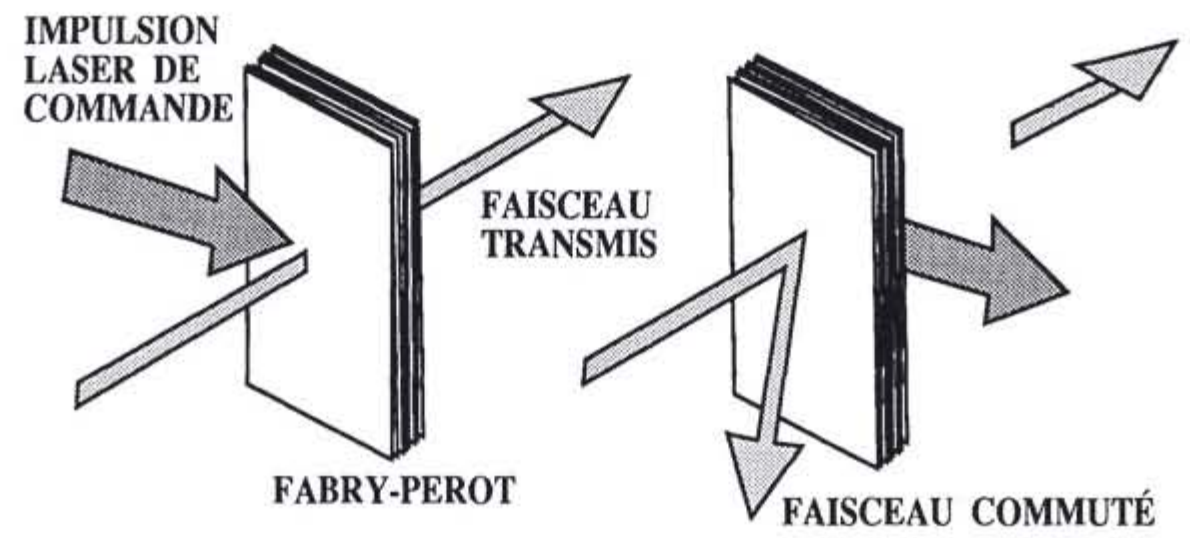

Figure 19 : Schéma de principe d'une porte optique commandée par un faisceau optique, basée sur le changement d'indice du Fabry-Perot non-linéaire

Quant aux temps de commutation, tout dépend de la non-linéarité optique impliquée et du temps de réponse intrinsèque du Fabry-Perot. Ce dernier est fonction de l'épaisseur du dispositif et de la finesse, c'est à dire du nombre moyen d'aller et retour dans la cavité. On n'a guère avantage à réduire la finesse mais par contre, en utilisant un milieu fortement non-linéaire et dense, on peut diminuer l'épaisseur nécessaire du Fabry-Perot. Les semiconducteurs sont un bon choix pour le milieu intracavité. Les non-linéarités peuvent y être d'origine thermique (lent), électronique (création d'un plasma) ou Kerr. Dans le cas de l'excitation d'un plasma, la création est aussi rapide que l'impulsion lumineuse est brève, mais la disparition des modifications est liée au temps de vie des porteurs. Pour une excitation virtuelle comme l'effet Stark optique, la non-linéarité ne dure que le temps du passage de l'impulsion lumineuse. En jouant avec ces trois paramètres, épaisseur du Fabry-Perot, durée des impulsions lumineuses et nature de l'excitation, on arrive à des temps de commutation inférieurs à la picoseconde (Paye 1993), aussi bien pour l'ouverture (ou la fermeture) que pour le retour à l'état initial (figure 20).

\section{TESTS DE CIRCUITS PAR MÉTHODE ÉLECTRO-OPTIQUE}

\subsection{Intérêt de la méthode optique}

Les années récentes ont vu un progrès continu dans le développement des dispositifs électroniques ultra-rapides et des circuits intégrés pour les ondes millimétriques. Ce progrès est tel qu'actuellement, les fréquences de coupure de tels systèmes sont bien au-delà de la gamme mesurable par les analyseurs de réseau conventionnels. En conséquence, les paramètres $\mathrm{S}$, qui servent à définir la réponse d'un composant, ne peuvent être déterminés dans la gamme des 
ondes millimétriques qu'en extrapolant les résultats obtenus à de plus basses fréquences grâce à un calcul basé sur la théorie des petits signaux. Rien ne prouve que cette méthode d'extrapolation soit valable pour prédire le comportement des dispositifs à plus haute fréquence.

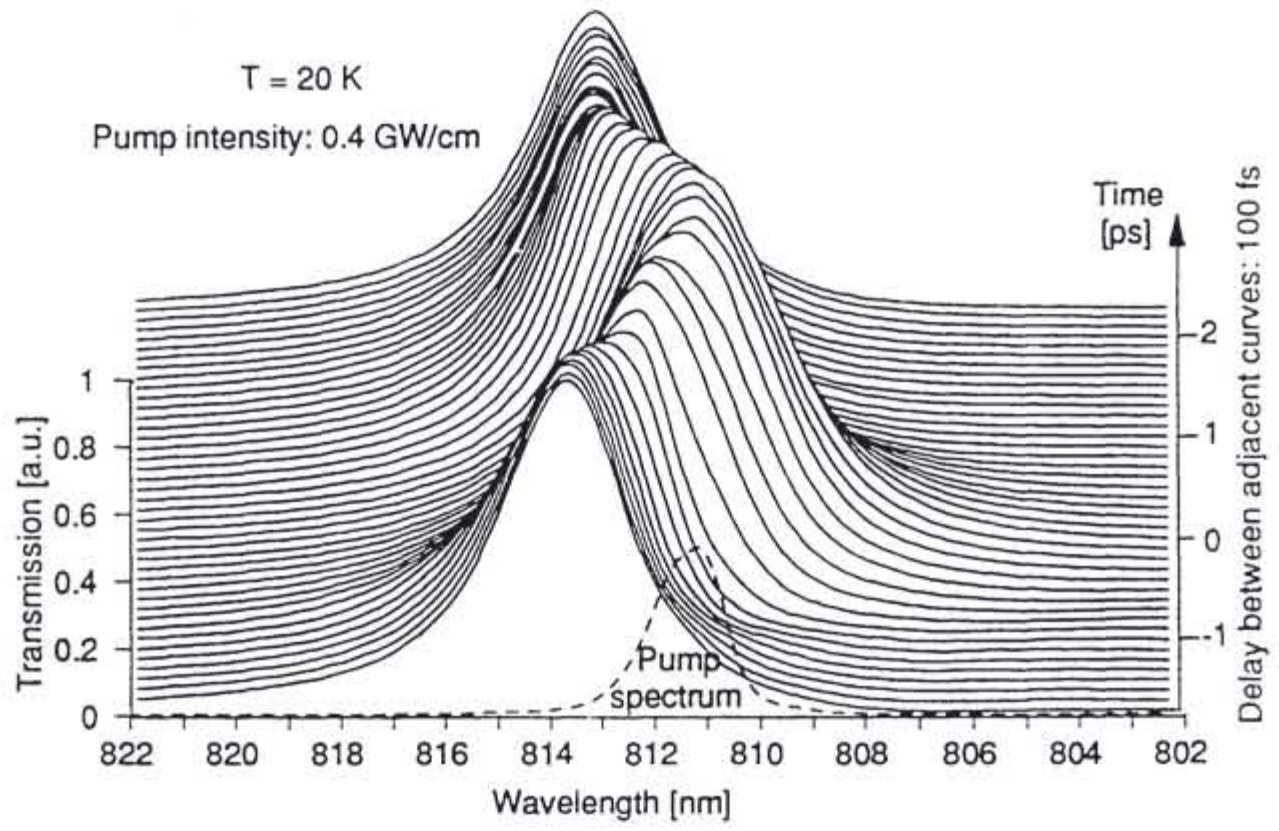

Figure 20 : Transmission résoluc en temps d'un étalon de Fabry-Perot traversé par une impulsion laser femtoseconde non absorbée par le milieu intra-cavité. Entre deux courbes il y a 100 femtosecondes. Le déplacement du pic de transmission vers les courtes longueurs d'onde se fait en moins d'une picoseconde et le retour à la position d'équilibre également. C'est la porte optique la plus rapide jamais démontrée pour l'aller et le retour

L'utilisation de méthodes de mesure dans le domaine temporel pour caractériser les dispositifs électroniques offre beaucoup d'avantages par rapport aux méthodes travaillant dans le domaine des fréquences. En mesurant la réponse du dispositif en fonction du temps, on peut obtenir sa réponse en fréquence en effectuant une transformation de Fourier. La méthode en fonction du temps offre l'avantage de pouvoir restreindre le signal à une fenêtre temporelle sélectionnée. On évite ainsi les réflexions et autres signaux indésirables qui perturbent habituellement les mesures. Cela permet entre autres de s'affranchir d'une partie des problèmes liés aux connections. Cependant l'utilisation de techniques temporelles pour caractériser les dispositifs a longtemps souffert du manque d'impulsions électriques vraiment courtes et de moyens de les mesurer avec exactitude. 


\subsection{Génération des impulsions électriques}

Les techniques de génération des impulsions électriques ultra-courtes reposent toutes sur le principe du commutateur photoconducteur. Des progrès importants ont été obtenus depuis les tous premiers travaux, mais le principe en est resté le même. La méthode originale a été proposée pour des commutations picosecondes par Auston (Auston, 1975) et Chi Lee (Lee, 1977).

Une ligne conductrice est déposée sur un substrat semi-conducteur choisi pour conduire très peu en l'absence d'excitation. Cette ligne est interrompue sur une courte distance et une tension est appliquée aux bornes. Une impulsion optique vient éclairer le semi-conducteur entre les électrodes; si l'énergie du photon est suffisante pour être dans une zone spectrale d'absorption correspondant aux transitions entre la bande de valence et la bande de conduction, la lumière génère une forte densité d'électrons et de trous libres et rend de ce fait le matériau conducteur.

La ligne métallique est ainsi "fermée" et un transitoire électrique est créé; il va se propager le long de la ligne (Frankel et al. 1991). La forme de ce transitoire dépend en partie de la durée de vie des porteurs photocréés, l'impulsion lumineuse étant supposée suffisamment brève pour que sa durée ne soit pas le facteur important. On a vu comment est actuellement choisi le substrat photoconducteur pour avoir une durée de vie brève (voir "Durées de vie").

De nombreux progrès ont été apportés depuis ces premiers travaux. Une partie porte sur la géométrie. La ligne de transmission interrompue est en effet source de nombreux effets indésirables. Cela se comprend en remarquant que tout transitoire électrique s'y propageant subira des réflexions diverses au niveau de l'interruption de ligne. De plus la propagation se fait mal dans cette géométrie. Actuellement on utilise souvent des lignes coplanaires, soit deux lignes métalliques parallèles et équivalentes, soit trois lignes, celle du centre étant plus fine que les deux autres.

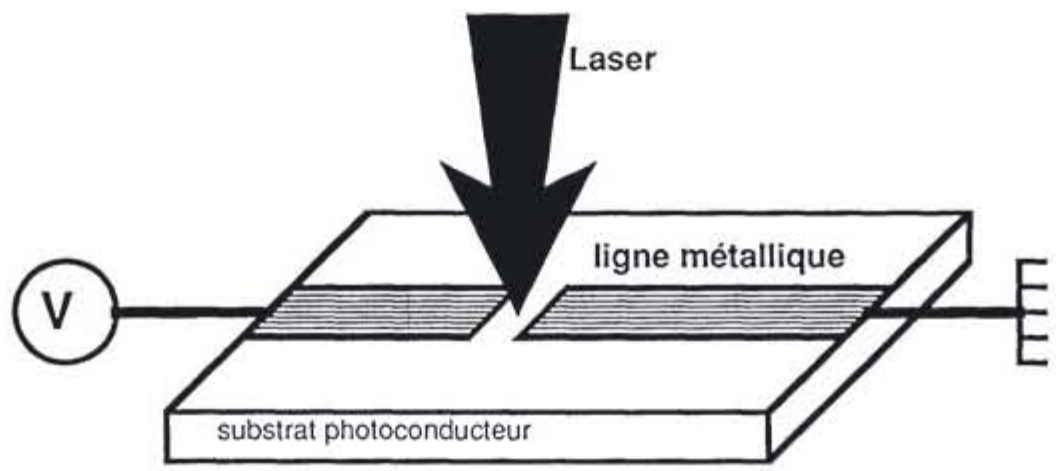

Figure 21: Schéma du dispositif expérimental servant à créer des impulsions électriques ultra-courtes. L'impulsion laser sert à créer un plasma d'électrons-trous qui conduit et ainsi ferme la ligne.

La génération du transitoire électrique peut se faire en envoyant l'impulsion optique entre deux lignes coplanaires portées à des potentiels différents (géométrie dite du contact glissant car la tache d'excitation peut se déplacer le long des lignes) (Grischkowsky et al., 1988), situation sans capacité ou inductance parasite. Une excitation dans une telle configuration crée une nouvelle distribution du champ par rapport aux deux lignes. Les impulsions électriques obtenues sont montrées dans la figure 22.Le temps de montée est de 340 femtosecondes, principalement déterminé par le temps de réponse de la détection. Le temps de redescente est nettement inférieur à la picoseconde. 


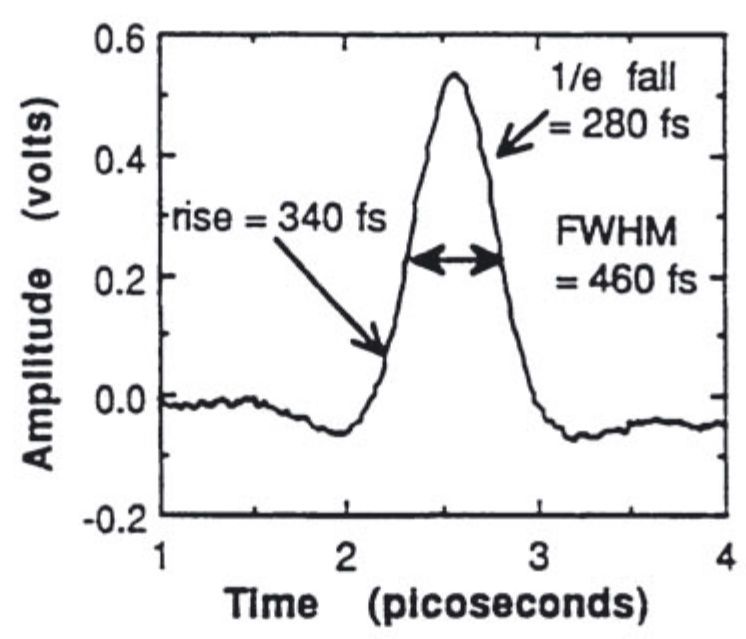

Figure 22 : Impulsion électrique générée par une impulsion optique femtoseconde envoyée sur un commutateur fabriqué sur un substrat de GaAs épitaxié à basse température. (d'après Gupta et al., 1991) droits réservés.

\subsection{Détection des impulsions électriques}

\subsubsection{Méthodes d'autocorrélation}

La première méthode suggérée a été d'utiliser pour la détection le même type d'interrupteur photoconducteur (gap) que pour la création. Le principe en est le suivant : sur une ligne métallique déposée sur un substrat photoconducteur, on intercale non plus un mais deux gaps qui bloquent la conduction de la ligne. Une des extrémités de la ligne est portée à un potentiel non nul.

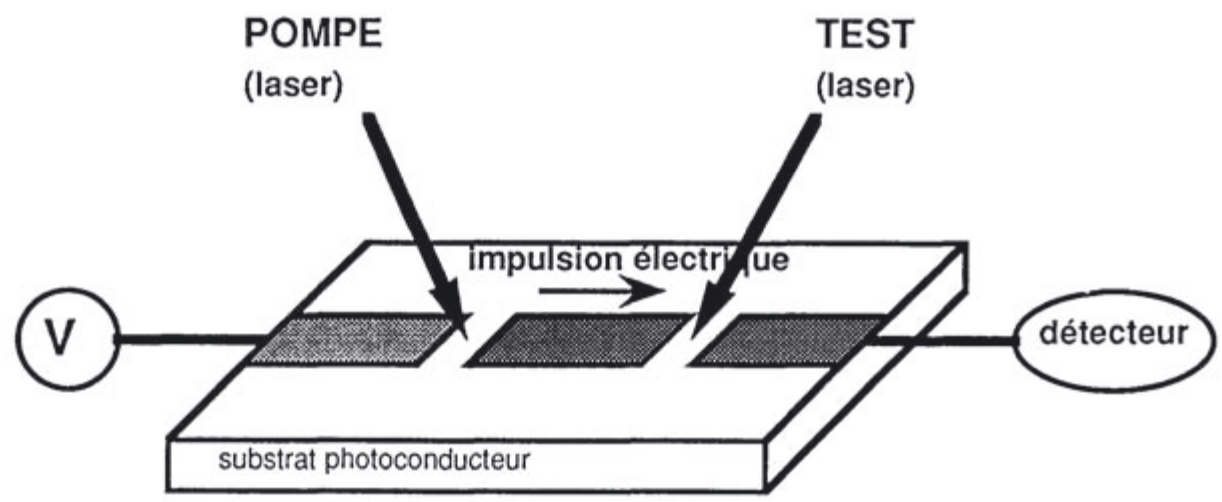

Figure 23 : Schéma de principe pour la génération d'une impulsion électrique et sa détection par la méthode d'autocorrélation. 
Une première impulsion optique vient court-circuiter le premier gap en créant des électrons et des trous libres dans le semiconducteur à l'intérieur du gap. Schématiquement, la deuxième partie de la ligne se trouve soudainement au potentiel de la première partie et cette "information" se propage et arrive au deuxième gap. Si ce deuxième commutateur est ouvert, cette information ne passe pas et aucun signal n'est recueilli à l'extrémité de la troisième partie de la ligne. Si maintenant une deuxième impulsion vient fermer le commutateur, on voit que le signal recueilli dépendra du moment où la deuxième impulsion optique arrive sur le deuxième gap, après ou avant l'arrivée du signal sur la ligne. Comme on sait faire varier de façon contrôlée le délai entre les deux impulsions, on peut remonter à la fonction d'autocorrélation de la fermeture des commutateurs et donc à l'impulsion électrique (Grischkowsky et al., 1988).

\subsection{2 Échantillonnage par effet électro-optique}

L'échantillonnage électro-optique nécessite l'utilisation d'un matériau qui ait une réponse électro-optique, c'est-à-dire que la présence d'un champ électrique y induit une biréfringence qui dépend de ce champ électrique.

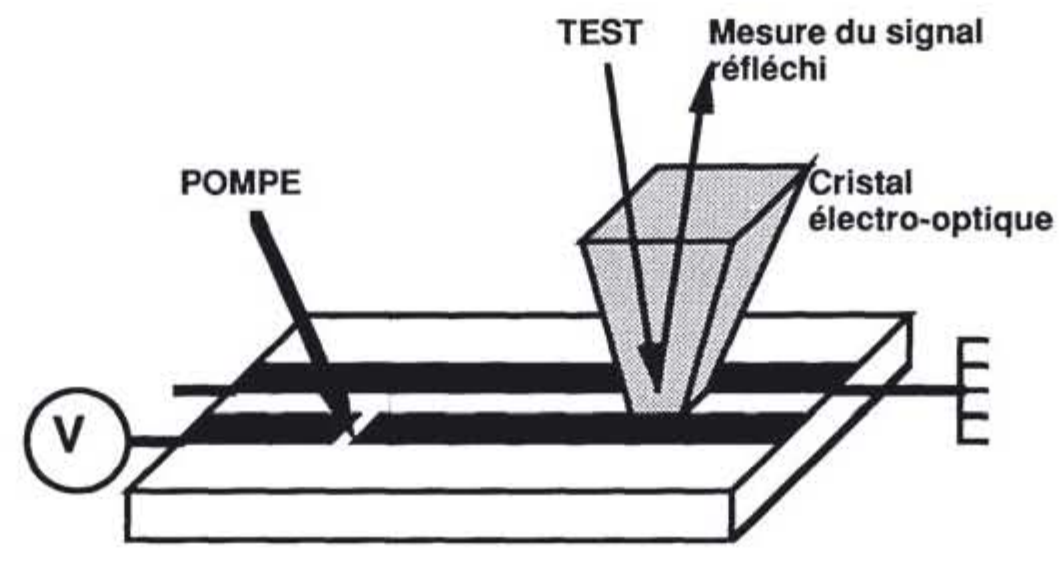

Figure 24 : Principe de la génération d'une impulsion électrique ultra-courte en géométrie de lignes coplanaires et détection de l'amplitude du champ électrique entre les lignes par un cristal électro-optique. En faisant varier le retard entre la pompe et le test, on échantillonne le signal électrique créé par la pompe sur le commutateur photoconducteur et propagé par les lignes coplanaires.

La technique d'échantillonnage électro-optique externe (J.A. Valdmanis, 1986, 1987 et 1990) emploie un tout petit cristal isolant et biréfringent pour mesurer le champ électrique, ceci afin de remonter aux différences de potentiel. Le schéma du système de mesure est montré sur la figure 24. La sortie d'un laser femtoseconde est divisée en deux, si bien que deux faisceaux identiques sont disponibles, l'un pour l'excitation de l'impulsion électrique, l'autre pour sa détection. Le premier sert à créer des porteurs dans un commutateur photoconducteur, générant ainsi une impulsion électrique courte. Le faisceau d'analyse est dirigé jusqu'à l'endroit de la mesure en utilisant une fibre optique flexible et là passe à travers divers éléments optiques et le cristal biréfringent. La polarisation du faisceau de test est modulée par le champ électrique de la ligne coplanaire qui interagit sur le cristal (effet Pockels). En variant le temps d'arrivée de l'impulsion d'excitation qui génère le transitoire électrique, l'impulsion laser d'analyse peut donc tester la forme temporelle de l'impulsion électrique lorsqu'elle passe au niveau du cristal électro-optique (KDP, BiSO3).

Puisque le faisceau laser de test peut être focalisé sur une tache de moins de $5 \mu \mathrm{m}$ de diamètre sur la face inférieure du cristal, la résolution spatiale de cette méthode est très bonne. De plus, aucun contact avec le circuit n'est nécessaire et de ce fait aucune charge n'est enlevée 
au circuit électrique. Avec ce système de mesure, des transitoires électriques très brefs, avec des spectres très larges, peuvent être analysés pour connaître le signal à l'entrée d'un dispositif à étudier, les réflexions sur l'entrée et le signal de sortie. Puisque le milieu électro-optique est extérieur au circuit électrique, cette technique ne nécessite pas de propriétés optiques spéciales du circuit et de ce fait peut être appliquée à beaucoup de dispositifs électroniques.

La résolution temporelle de cette technique est contrôlée par la durée de l'impulsion optique, car c'est elle qui sert de porte temporelle dans l'échantillonnage électro-optique. On utilise généralement des impulsions de 100 femtosecondes ou moins. Il existe d'autres limitations, mais cela donne des mesures électro-optiques (Jackson et al., 1992) valables au moins jusqu'à $1 \mathrm{THz}$.

\subsection{Conclusion}

Il est possible d'utiliser la technique de l'échantillonnage électro-optique associée à des lignes de transmission coplanaires pour caractériser la réponse temporelle de dispositifs ultra-rapides. Cette technique permet d'étendre à de plus hautes fréquences les mesures faites avec des méthodes plus classiques. Elle possède de plus un énorme avantage, c'est d'être la seule à pouvoir faire les études dans le régime non-linéaire des forts signaux. En conclusion, cette approche s'avère de plus en plus indispensable pour étudier des temps de réponse de dispositifs qui deviennent comparables aux temps mêmes d'évolution du système électronique à l'intérieur du dispositif.

\section{LES FEMTOSECONDES EN CHIMIE ET EN BIOLOGIE}

\subsection{Introduction}

La vision macroscopique de la chimie, qui est une première approche de ce domaine, fait place à une vision à la fois plus microscopique et plus synthétique lorsque les connaissances progressent. En particulier, les réactions chimiques dans leur diversité sont gouvernées par les mêmes mécanismes fondamentaux, à savoir les vibrations, les changements de conformation et les transferts de charge.

Traiter les mécanismes fondamentaux, c'est regarder les processus à une échelle de petite taille et cela implique de ce fait de regarder les phénomènes sur une très courte échelle de temps qui est souvent celle des femtosecondes. Par exemple, dans le cas de la séparation de deux fragments, la liaison moléculaire n'existe plus dès que leur distance est de l'ordre de l'Angström; pour des vitesses de séparation raisonnables de $1000 \mathrm{~m} / \mathrm{s}$, cela correspond à un temps de $100 \mathrm{fs}$. De 0 à $100 \mathrm{fs}$, le système est dans un état non stationnaire dit de transition, qu'il est particulièrement intéressant d'étudier par spectroscopie femtoseconde.

Dans le domaine des macromolécules et de la biologie, il y a des avantages supplémentaires à utiliser des impulsions courtes. Par exemple, dans l'excitation sélective d'un chromophore, on tire parti de la diffusion lente de la chaleur dans les macromolécules, ce qui évite une dispersion de l'excitation. Il y a également une simplification des problèmes que posent ces molécules complexes : dans un temps court, peu d'événements surviennent, même pour les molécules compliquées. On fait alors un découpage en tranches simples de phénomènes complexes.

Enfin le terme de systèmes réactifs intelligents peut être évoqué. Beaucoup d'espoirs sont placés sur la maîtrise du couplage entre la cohérence des systèmes atomiques et moléculaires et la lumière. On espère alors guider les réactions et les orienter dans une direction choisie et intéressante.

\subsection{Dynamique d'un paquet d'ondes}

L'excitation d'une molécule peut avoir lieu par l'absorption d'un photon qui fait passer un 


$$
\overline{I I}
$$


une partie des électrons franchit le seuil par effet tunnel et rapidement le paquet d'ondes disparaît.

La mesure de ces oscillations donne accès aux effets d'une cage de solvant. Si la molécule considérée est entourée de molécules de solvant qui forment une sorte de cage, cela freine la dissociation. Cela se traduit par des oscillations du paquet d'ondes là où elles n'étaient pas attendues pour une molécule nue, mais celles-ci disparaissent vite car la modification de la conformation de la molécule excitée perturbe un peu plus à chaque oscillation la cage qui finit par éclater (Liu, 1993).
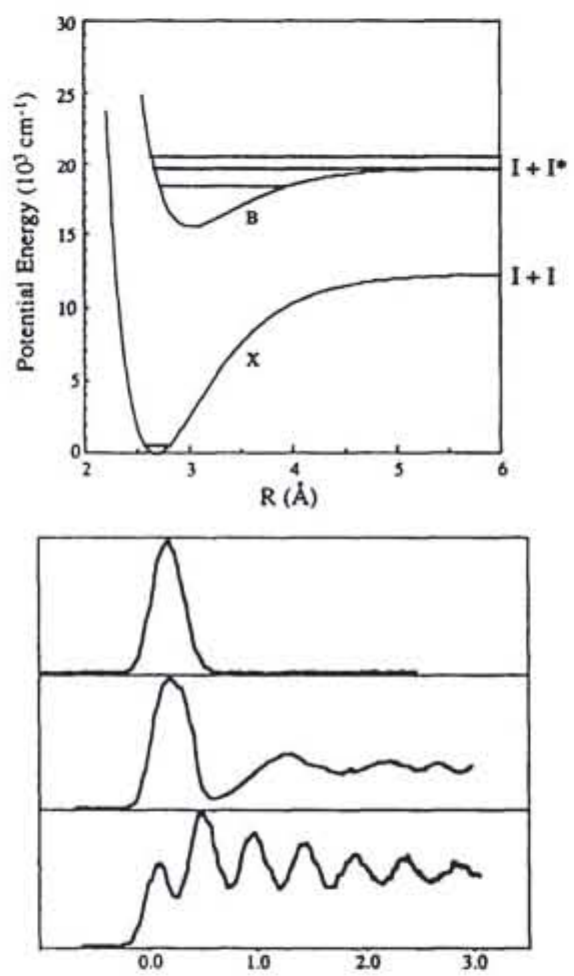

Temps (ps)

Figure 26 : Dynamique du paquet d'ondes (partie inférieure de la figure) pour trois énergies d'excitation (partie supérieure de la figure) dans la dissociation de la molécule d'iode. D'après Zewail, 1994.

Droits réservés.

Ces oscillations de paquet d'ondes ont été observées à des échelles de temps de quelques dizaines ou quelques centaines de femtosecondes. Cela correspond à la visualisation en temps direct du mouvement du noyau pendant une réaction chimique. Elles ont été observées sur des molécules alcalines simples en phase gazeuse, où la relaxation des modes vibrationnels est faible. C'est également vrai pour des molécules simples dans les liquides où il y a un déphasage par le bain. Plus récemment, elles ont été observées dans des macromolécules très complexes, les centres réactionnels bactériens (Vos et al., 1993). Dans ce dernier cas, seuls quelques modes de vibration sont activés et sont peu amortis. Dans ce cas, la réduction des degrés de liberté 
(2D) liée à la présence d'une bicouche lipidique (la membrane de la cellule) réduit la dissipation du mouvement.

Pendant les oscillations ou la dissociation, il y a changement progressif des longueurs d'onde pouvant interagir avec la molécule, à cause du caractère non-harmonique de la surface de potentiel. Pour que le laser agisse de façon continue, il faudrait donc faire varier ses longueurs d'onde de façon contrôlée pendant l'impulsion. On peut aussi penser à des impulsions répétitives à la cadence des oscillations. Tout ceci est à portée d'expérience car le contrôle et le formage des impulsions lasers ont nettement progressé. Le concept de contrôle moléculaire n'est plus une utopie.

\subsection{Dynamique des molécules complexes}

La vision, l'absorption chlorophyllienne ou l'oxygénation de l'hémoglobine sont des processus essentiels pour la vie. Leur étude n'est pas simple car les molécules impliquées sont complexes. Toutefois, dans chacun des exemples cités plus haut, il existe une étape fondamentale qui est la base du déclenchement du processus complet. Cette première réaction (réaction au sens large) se passe en des temps très brefs et grâce à cette particularité, elle a lieu alors que la suite du processus est encore gelée. Travailler avec des impulsions femtosecondes permet d'étudier cette réaction primaire. Par exemple, il a été montré que le changement de conformation de l'hème (site actif de l'hémoglobine) a lieu en moins de 300 fs après le décrochement de l'oxygène (Martin et al., 1983). De même l'étude des premières étapes de la vision ou de l'absorption chlorophyllienne permet de comprendre pourquoi le rendement est si bon, meilleur que ce qu'on sait faire avec une technologie à base de silicium ou d'un matériau apparenté.

\subsection{Solvatation d'électrons}

Une réaction d'oxydoréduction correspond à un échange d'électrons. Or une partie des réactions se passe en milieu aqueux. L'eau est un milieu très polarisable dont la réponse en face de l'électron échangé est importante. Il est donc judicieux d'étudier le processus de solvatation de l'électron dans l'eau pour comprendre le comportement dynamique de l'eau.

Une première impulsion laser crée un électron libre dans un milieu non aqueux contenu dans une micelle. Dès qu'il franchit la membrane, il est entouré par les molécules d'eau polarisées. Les étapes primaires de la capture d'un électron ont été mises en évidence (Gauduel et al., 1989). En particulier l'apparition d'une espèce insoupçonnée a été démontrée, il s'agirait d'un électron pré-solvaté. Le couplage primaire entre l'électron et l'eau interfaciale s'effectue en 140 fs et se poursuit par la relaxation d'un état électronique transitoire d'une durée de vie de $270 \mathrm{fs}$.

Les mécanismes radicalaires ultra-rapides ont une dynamique réactionnelle fortement influencée par des gradients transitoires locaux (densité de liaison hydrogène, champ coulombien intense). Leur étude théorique nécessite de considérer la forme et les caractéristiques des puits de potentiel associés aux espèces réactives, en incluant la position de l'électron et sa quantité de mouvement quantique.

\section{LES ATOMES EN CHAMP SUPER-INTENSE}

\subsection{Introduction}

L'amplification d'impulsions lumineuses dans des cristaux à très haute énergie de saturation (plusieurs Joules par $\mathrm{cm}^{2}$ ) comme le Saphir dopé au Titane, l'Alexandrite ou les cristaux de $\mathrm{LiCaF}$ permettent de gagner plusieurs ordres de grandeur par rapport aux colorants et ceci grâce aux techniques héritées des radaristes. Ces nouveaux amplificateurs vont permettre d'obtenir des énergies de plusieurs Joules pour des impulsions de 30 à $50 \mathrm{fs}$. 
Avec l'optimisation de la focalisation de ce type d'impulsion lumineuse, on peut espérer atteindre des intensités comprises entre $10^{19}$ et $10^{22} \mathrm{~W} / \mathrm{cm}^{2}$. L'interaction lumière - matière obtenue avec ce type d'installation est complémentaire de celle obtenue avec de très lourdes installations avec l'avantage pour les systèmes femtosecondes d'avoir entre autres une récurrence nettement plus élevée.

On sait générer des champs lumineux intenses dépassant l'unité atomique d'intensité (3.5

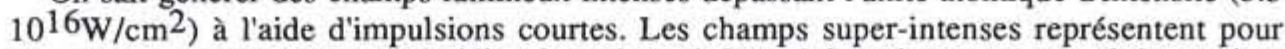
l'atome un environnement inédit, dans lequel les phénomènes connus doivent être profondément modifiés. En effet, à une intensité d'une unité atomique, l'amplitude de la composante électrique du champ électromagnétique est égal au champ du proton sur la première orbite de Bohr de l'atome d'hydrogène. L'électron est soumis de façon égale au champ coulombien et au champ électromagnétique, et toutes les images traditionnelles, qui considèrent le champ électromagnétique comme une perturbation faible, doivent être abandonnées. Pour les très fortes intensités $\left(10^{20} \mathrm{~W} / \mathrm{cm}^{2}\right)$, la situation est complètement inversée, et c'est le champ du proton qui peut être considéré comme une perturbation pour l'électron dans le champ électromagnétique.

Au delà de l'intérêt qu'il y a à utiliser des impulsions ultra-brèves pour obtenir des champs super-intenses (aspect forte intensité), il est crucial d'utiliser des impulsions laser de courte durée (aspect temporel). En effet, il est impossible de placer un atome neutre dans un champ lumineux intense dont l'intensité montre progressivement car le phénomène d'ionisation se produit avant que l'intensité maximum de l'impulsion lumineuse soit atteinte. Ainsi, on ne peut espérer soumettre l'atome à une intensité supérieure à $10^{15} \mathrm{~W} / \mathrm{cm}^{2}$ avec des impulsions picosecondes.

Si on peut atteindre l'intensité maximum avant que le phénomène d'ionisation n'ait eu lieu, on étudie le phénomène d'ionisation mutiphotonique multiple directe dans lequel plusieurs électrons sont arrachés simultanément à l'atome. Très peu de choses sont encore connues sur ce processus. Des mécanismes d'oscillations collectives des couches atomiques entières, excitées de manière cohérente par le champ intense, ont été proposés. On a aussi évoqué de possibles excitations en couches internes, qui pourraient conduire à d'importantes applications concernant l'émission et l'amplification de lumière dans le domaine V.U.V. ou X mous.

\subsection{Génération d'harmoniques d'ordre élevé}

\subsubsection{Génération en champ intense}

La génération d'harmoniques d'une lumière intense et plus particulièrement d'un laser est un phénomène bien connu. Il a d'abord été observé dans un cristal (Franken, 1961) puis dans un gaz (Word et New, 1967). Le doublage de fréquence (harmonique 2) est couramment produit avec des cristaux comme le KDP ou le BBO. Pour des raisons de symétrie, il n'y a pas d'harmoniques pairs dans un milieu centrosymétrique (hydrogène, gaz rares,...)

En présence d'une excitation lumineuse intense sur des atomes, il y a génération d'harmoniques de la fréquence fondamentale du laser. En optique non-linéaire classique (théorie des perturbations), il est prévu que l'harmonique d'ordre $q$ ait une intensité proportionnelle à Iq. Le facteur de proportionnalité est très faible et décroît fortement lorsque l'ordre $q$ augmente. Pour des intensités moyennes, les harmoniques obtenus sont bien décrits par cette théorie conventionnelle.

Cependant autour de $10^{13}$ à $10^{14} \mathrm{~W} / \mathrm{cm}^{2}$, il apparaît des distorsions par rapport aux résultats classiques attendus. Le moment dipolaire induit a beaucoup de composantes qui sont des harmoniques élevés de la fréquence du laser excitateur. Alors qu'à quelques $10^{12} \mathrm{~W} / \mathrm{cm}^{2}$ l'intensité de l'harmonique subit une décroissance importante lorsque $q$ augmente, elle reste sur un plateau de valeur constante à partir du 5 ème harmonique dès $10^{13} \mathrm{~W} / \mathrm{cm}^{2}$ avant de redécroître brusquement pour des ordres élevés. Cette dernière coupure dépend de l'intensité du laser et de la nature de l'atome. La dépendance en éclairement pour un ordre donné $q$ ne suit plus la loi perturbative qui prédit une dépendance en I9. Par exemple, pour l'harmonique 17 du Xénon, on 
trouve une pente 8 et non 17 et ceci pour des intensités lumineuses bien inférieures aux valeurs nécessaires pour l'ionisation (Balcou P., 1993) La génération d'harmoniques élevés est un phénomène essentiellement non-perturbatif.

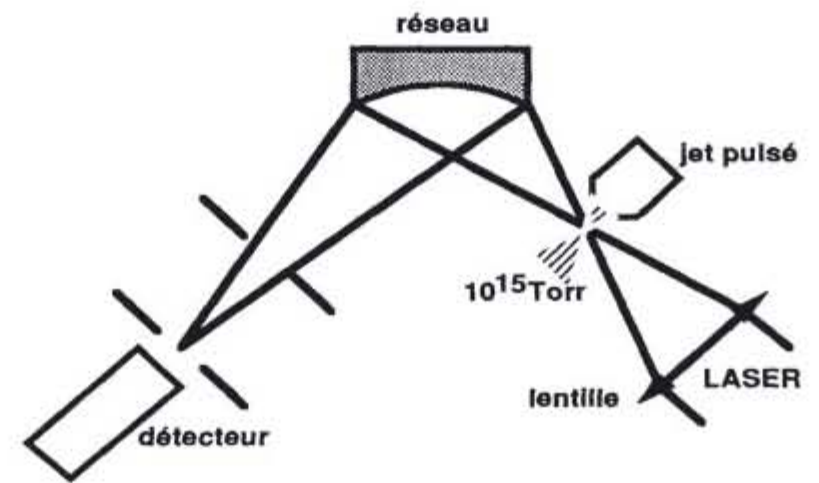

Figure 27 : Production et mesures des harmoniques de la fréquence fondamentale du laser par focalisation dans un jet atomique pulsé.

Ce processus a été modélisé en résolvant l'équation de Schrödinger dépendante du temps. En plus du hamiltonien atomique, il y a un terme d'interaction avec le champ électromagnétique oscillant à la fréquence du laser. Cette théorie reproduit l'existence du plateau d'harmoniques et la dépendance de la coupure avec l'intensité du laser.

Pour tenter de comprendre l'origine de ces harmoniques d'ordre élevé, on peut aussi considérer la dynamique d'un électron dans un potentiel anharmonique confinant :

$$
\mathrm{V}(\mathrm{x})=\frac{1}{2} \omega_{0}^{2} \mathrm{x}^{2}+\frac{1}{4} \quad \mathrm{v} \mathrm{x}^{4}
$$

et soumis à une force périodique de basse fréquence. $x$ est la position de l'électron et $v$ un paramètre. $\omega_{0}$ sera égale à la fréquence de l'atome. On se place dans la situation expérimentale où la longueur d'onde du laser est dans le visible (ou proche) et où l'atome a son seuil d'absorption haut en énergie (gaz rares par exemple); le milieu est transparent pour le laser. L'équation du mouvement est :

$$
\frac{d^{2} x}{d t^{2}}+\Gamma \frac{d x}{d t}+\omega_{0}^{2} x+v x^{3}=\text { Elaser } \sin \omega \text { laser } t
$$

$\Gamma$ est un coefficient d'amortissement et $\omega_{0} \gg \omega_{\text {laser. }}$ Cette équation n'admet pas de solution analytique et pour des forces d'excitation très importantes, le mouvement devient même chaotique. Le problème est traité numériquement et reproduit aussi les résultats expérimentaux sur les harmoniques.

L'examen de la position $x(t)$ permet d'avoir une vision de ce qui se passe. En tirant parti de la grande différence des fréquences, sur une courte échelle de temps, l'électron oscille surtout au fond du puits de potentiel confinant. Toutefois, le centre de gravité de cette oscillation se déplace à cause du champ de l'onde électromagnétique. En prenant en compte le terme d'amortissement, on voit que le mouvement de fond de puits se compose d'oscillations périodiquement excitées (pendant la partie de la période où l'électron est accéléré par le champ externe) puis amorties. Il est aisé de comprendre que cette trajectoire un peu compliquée va permettre de rayonner des harmoniques de la fréquence du laser excitateur.

De spectaculaires émissions d'harmoniques de rang très élevé ont déjà été observées, comme l'harmonique 125 d'un laser à $1053 \mathrm{~nm}$ dans l'hélium à 70 torrs ou l'harmonique 131 dans le 
néon à 40 torrs (Balcou Ph., Salières P. et L'huillier A., 1993). Cela correspond à des longueurs d'onde autour de $8 \mathrm{~nm}$, dans le domaine des rayons $\mathrm{X}$. Ce rayonnement est cohérent mais son intensité est pour l'instant encore trop faible (de l'ordre de $10^{5}$ photons pour les exemples ci-dessus) pour qu'il soit utilisé dans des applications.

La position de la coupure est donnée par:

$$
q_{\max } K \omega=I \mathrm{p}+3.2 \mathrm{U}_{\mathrm{p}}
$$

Ip est le potentiel d'ionisation de l'atome et $\mathrm{U}_{\mathrm{p}}$ le potentiel pondéromoteur, c'est à dire l'énergie cinétique moyenne d'oscillation d'un électron libre. Nous reviendrons plus loin sur ce potentiel pondéromoteur. L'équation ci-dessus indique que la position de la coupure en fréquence dépend bien de l'atome et de l'intensité du champ électromagnétique.

\section{2 .2 Génération d'harmoniques en champ super-intense}

A des régimes supérieurs, on s'attend à de nouveaux phénomènes où la modification de la réponse de l'électron au champ électrique oscillant du laser ne vient plus de son potentiel confinant autour du noyau, mais de la composante magnétique du champ de l'onde optique. Dans l'interaction d'une charge avec le champ électromagnétique:

$$
\vec{F}=e \vec{E}+\frac{e}{c} \vec{V} \wedge \vec{B}
$$

le deuxième terme est souvent négligé, mais ce n'est plus possible aux forts champs considérés ici.

rayonnement $\omega$
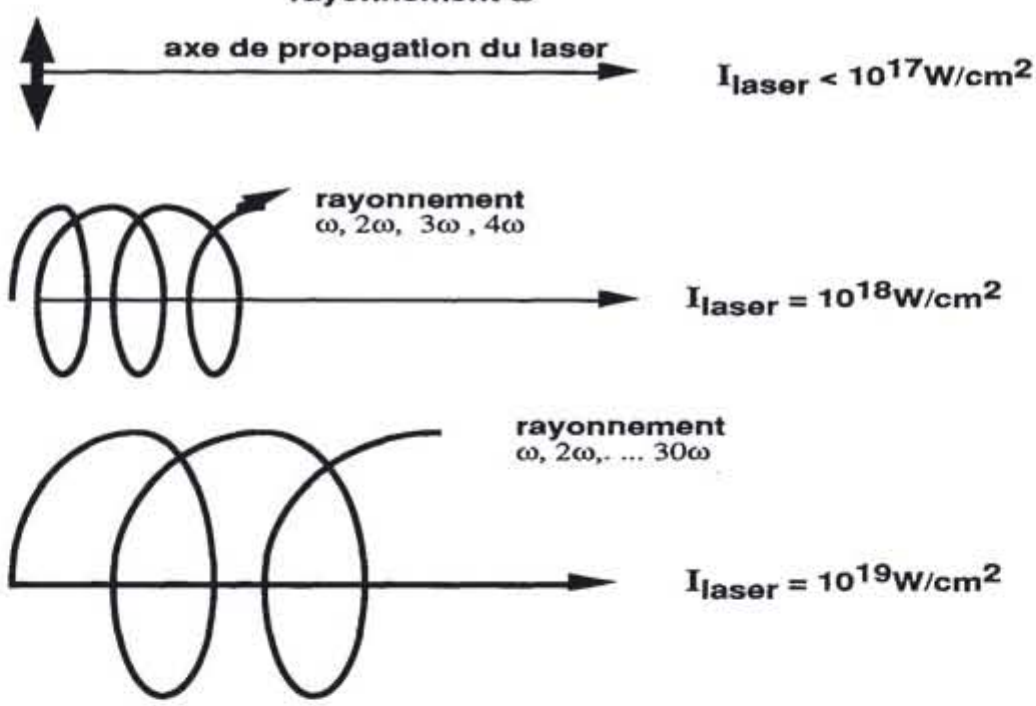

Figure 28: Mouvement d'un électron relativiste en présence d'un champ laser ultra-intense. Le dipôle, soumis à un champ laser moyen, rayonne dans la direction de propagation du laser dans le cas d'une assemblée de dipôles. Son rayonnement se transforme lorsque le champ augmente et acquiert des composantes dont les directions se situent sur des cônes centrés sur la direction du laser. D'après G. Mainfray , communication privée. Droits réservés.

Avec ces électrons relativistes, il est prévu d'atteindre l'harmonique 1000 pour des puissances de $10^{20} \mathrm{~W} / \mathrm{cm}^{2}$. 


\subsection{Ionisation des atomes en champ laser intense}

\subsubsection{Rappel sur le potentiel pondéromoteur}

L'énergie pondéromotrice $U_{\mathrm{p}}$ correspond à l'énergie cinétique moyenne d'un électron libre initialement au repos oscillant dans un champ électromagnétique :

$$
\mathrm{m} \frac{\mathrm{dv}}{\mathrm{dt}}=-\mathrm{eE} \cos \omega \mathrm{t}
$$

Si la contribution magnétique et les variations spatiales sont négligées, l'énergie cinétique moyenne, donc définie sur au moins une période, est :

$$
\frac{1}{2} \mathrm{~m}<\mathrm{v}^{2}>_{\mathrm{temps}}=\frac{\mathrm{e}^{2} \mathrm{E}^{2}}{4 \mathrm{~m} \omega^{2}}=\mathrm{U}_{\mathrm{p}}
$$

L'expression ci-dessus donne le déplacement de l'énergie d'un électron libre dans le champ du laser. Pour un électron sur une orbitale atomique liante, il faut considérer différemment les orbitales profondes et les états de Rydberg. Le déplacement de l'état fondamental (petite orbitale) est pratiquement nul dans le champ du laser. Par contre, pour les grands nombres quantiques, c'est-à-dire pour les états près du seuil d'ionisation, la trajectoire de l'électron est très grande et celui-ci passe très peu de temps près du noyau. Dar conséquent il est sensible à la perturbation du laser et se comporte pratiquement comme un électron libre. Le déplacement en énergie des états de Rydberg est égale au potentiel pondéromoteur.

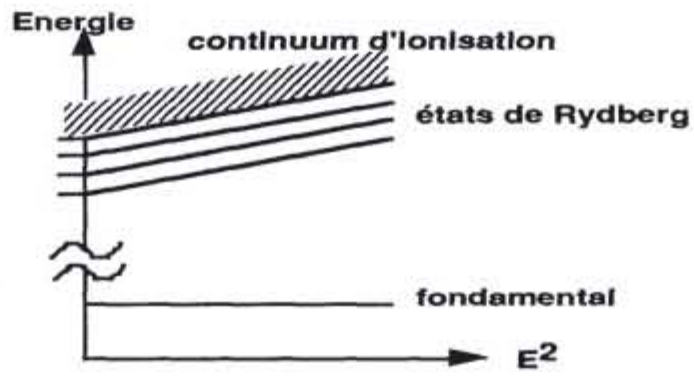

Figure 29 : Représentation du déplacement des niveaux d'énergie en présence d'un champ laser intense. Les états de Rydberg et les états đu continuum d'ionisation se déplacent tous de la même quantité qui est le potentiel pondéromoteur.

\subsubsection{Techniques de mesure de l'ionisation}

Lorsqu'un atome est soumis à un champ électromagnétique intense, un électron peut être arraché sous l'action du champ électrique et laisser un ion derrière lui. La spectrométrie de masse/charge des ions émis permet de déterminer le degré d'ionisation atteint. Les spectres des électrons émis permettent une analyse plus fine du processus car ils donnent des renseignements sur l'état final de celui-ci. Certaines composantes du spectre d'électron peuvent aussi signaler des ions laissés par le processus d'ionisation dans un état excité d'où ils peuvent éventuellement rayonner.

\subsubsection{Ionisation par un laser extrêmement intense}

Dans le cas d'un laser très intense, la meilleure description de l'action de l'onde sur l'atome se fait non pas en termes de photons mais en considérant classiquement l'interaction avec le champ électrique oscillant de la lumière. L'attraction du noyau représente pour l'électron lié un potentiel 
confinant et il faut fournir l'énergie d'ionisation pour faire sortir l'électron de ce puits en l'absence de champ.

En présence d'un champ électrique, il faut ajouter un terme en -eEx au potentiel, terme qui abaisse la barrière de potentiel d'un côté et le relève de l'autre. Si cet abaissement est suffisant, l'électron peut sortir du puits de potentiel dû à l'atome. Comme le champ est oscillant, la direction de sortie s'inverse régulièrement. Cet électron qui a échappé à l'attraction du noyau peut cependant revenir vers lui si il ne s'est pas suffisamment éloigné avant que le sens du champ électrique ne s'inverse. Par collision, il peut alors ioniser à nouveau l'ion qu'il avait quitté. C'est une ionisation multiple séquentielle.

\subsubsection{Ionisation par un laser très intense}

Un champ électrique abaisse l'énergie de sortie de l'électron hors du potentiel attractif du noyau. Si l'abaissement provoqué par le champ de l'onde lumineuse est insuffisant pour une sortie directe comme ci-dessus, il reste la possibilité de traverser par effet tunnel la barrière dont l'épaisseur et la hauteur ont diminué. Le critère pour que ce mécanisme se produise est que le "temps tunnel" (l'inverse de la probabilité de sortie par effet tunnel) soit plus court que la période du laser. En effet, si le champ électrique oscille plus rapidement que l'électron ne se décide à sortir, le sens du champ électrique s'inverse et tout est à recommencer de l'autre côté!

Cet effet n'est pas la seule source d'ionisation à champ fort. Les électrons de valence (faiblement liés) accomplissent sous l'effet du champ laser un mouvement d'oscillations au cours duquel ils peuvent atteindre des vitesses relativistes, et dont l'amplitude excède largement la taille de l'atome. Par contre les électrons du coeur, fortement liés, ne sont pas directement affectés par le champ. Néanmoins, au cours de leur oscillation, les électrons externes entrent en collision avec le coeur, produisant de l'ionisation, simple ou multiple, collisionnelle. Ce mécanisme explique des probabilités d'ionisation en couche interne beaucoup plus grandes que le modèle à particules indépendantes ne le prévoit.

\subsubsection{Ionisation par un laser assez intense}

A des intensités modérées, le concept de photon redevient pertinent. En principe, un atome de Xénon absorbe sept photons à $620 \mathrm{~nm}$ pour atteindre son continuum d'ionisation. Les spectres d'énergie des électrons émis montrent une série de pics séparés par l'énergie d'un photon, ce qui permet de conclure que l'électron peut continuer à absorber des photons en nombre supérieur à celui strictement nécessaire pour l'ionisation (Agostini, 1979). Ce phénomène a été nommé "ionisation au delà du seuil" (IAS, en anglais ATI pour "above threshold ionisation"). L'énergie cinétique de l'électron émis doit être :

$$
E_{\text {cinétique }}=\mathrm{n} \quad \boldsymbol{h} \quad \omega-\text { E }_{\text {ionisation }}
$$

Ces absorptions multiphotoniques n'apparaissent que pour des champs électromagnétiques incidents assez intenses. Ceci implique en parallèle la distorsion du spectre d'énergie de l'atome par effet Stark dynamique. Cette distorsion est importante sur le continuum d'ionisation dont I'énergie augmente d'une quantité qui est potentiel pondéromoteur (voir figure ci-dessus). Rappelons que ce potentiel n'est rien d'autre que l'énergie d'oscillation de l'électron libre dans le champ électromagnétique intense. Bien que sa valeur soit importante, $\left(3.4 \mathrm{eV}\right.$ à $\left.10^{14} \mathrm{~W} / \mathrm{cm}^{2}\right)$, il n'avait jamais été observé avant 1988.

La raison en était que les impulsions optiques utilisées jusque là étaient de durée trop longue ce qui permettait à l'électron libre de sortir latéralement du faisceau optique avant que celui-ci ne disparaisse. Or en traversant ce gradient de champ dirigé dans le sens du déplacement, l'énergie d'oscillation était transférée à l'énergie de translation qui est la quantité que l'on mesure.

La situation est bien différente avec des impulsions de $100 \mathrm{fs}$ car alors l'électron libre n'a pas le temps de sortir du faisceau laser avant que l'impulsion ne s'éteigne par l'arrière (et donc sans travail car le gradient de champ est perpendiculaire à la translation de lélectron) (Agostini et al., 1987 ; Freeman et al., 1987; Muller et al, 1988).

On observe alors un spectre de photoélectron décalé de ce potentiel pondéromoteur qui peut atteindre des valeurs supérieures à $5 \mathrm{eV}$ (Muller et al., 1988). Cela signifie que des pics attendus 
de photoélectrons disparaissent car le seuil d'ionisation augmente quand $\mathrm{U}_{\mathrm{p}}$ augmente; tous les autres (on en observe jusqu'à une dizaine) sont décalés de cette valeur $\mathrm{U}_{\mathrm{p}}$. Ce résultat est tout à fait conforme à la théorie. De plus, des sous-structures apparaissent dans les pics (Freeman et al., 1987). Elles sont dues au passage à résonance d'états intermédiaires. Ces spectres sont très riches et donnent beaucoup de renseignements sur les atomes soumis à un champ laser intense.

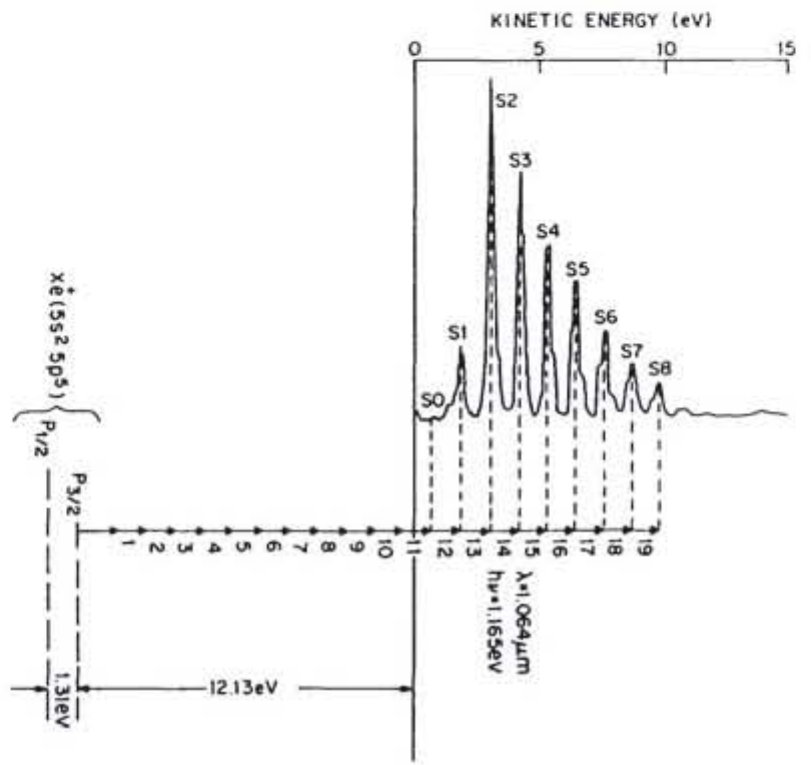

Figure 30 : Spectre de l'énergie cinétique d'un électron émis à partir d'un atome de Xénon. La longueur d'onde fondamentale du laser est $1.06 \mu \mathrm{m}$ et la durée des impulsions de l'ordre de $20 \mathrm{ps}$.(Freeman, 1986) Droits réservés.

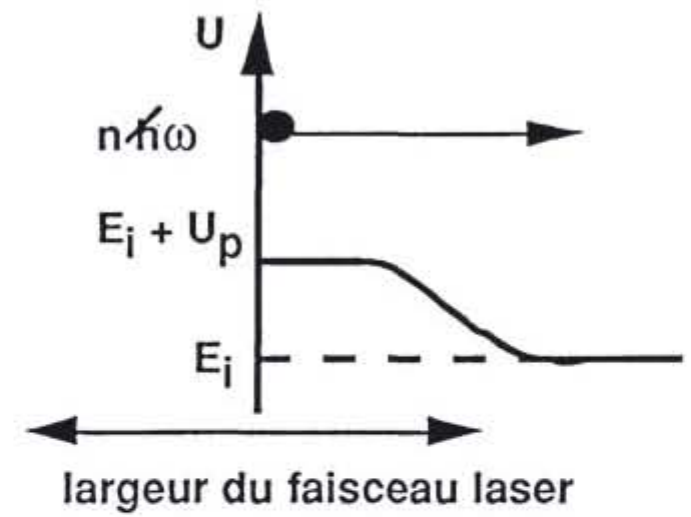

Figure 31 : Énergie de l'électron ionisé par une impulsion "longue", c'est-à-dire lorsque le champ optique dure plus longtemps que le temps mis par l'électron pour sortir du faisceau. Le potentiel qu'il perd $\left(\mathrm{U}_{\mathrm{p}}\right)$ est transformé en énergie cinétique (déplacement) qui passe de $n \hbar \omega-\left(E_{i}+U_{p}\right)$ a $n \hbar \omega-E_{i}$. 

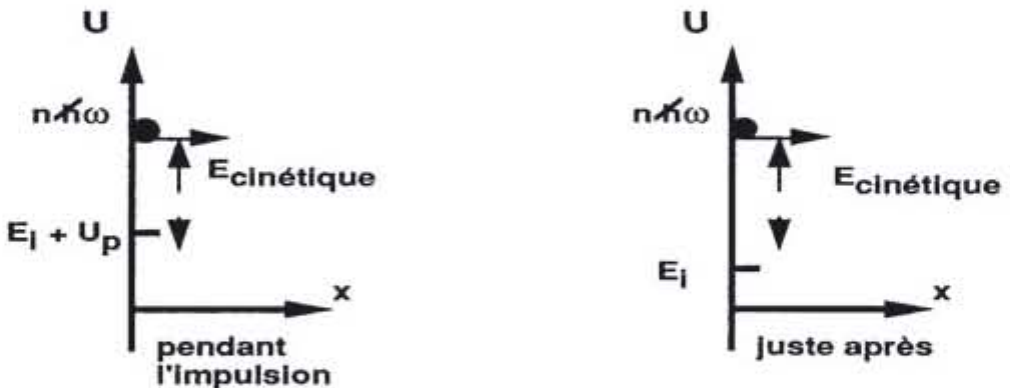

Figure 32 : Schéma de l'énergie d'un électron excité par n photons dans le champ d'une impulsion laser ultra-courte. L'électron garde une énergie cinétique qui reflète l'état de latome pendant l'impulsion.
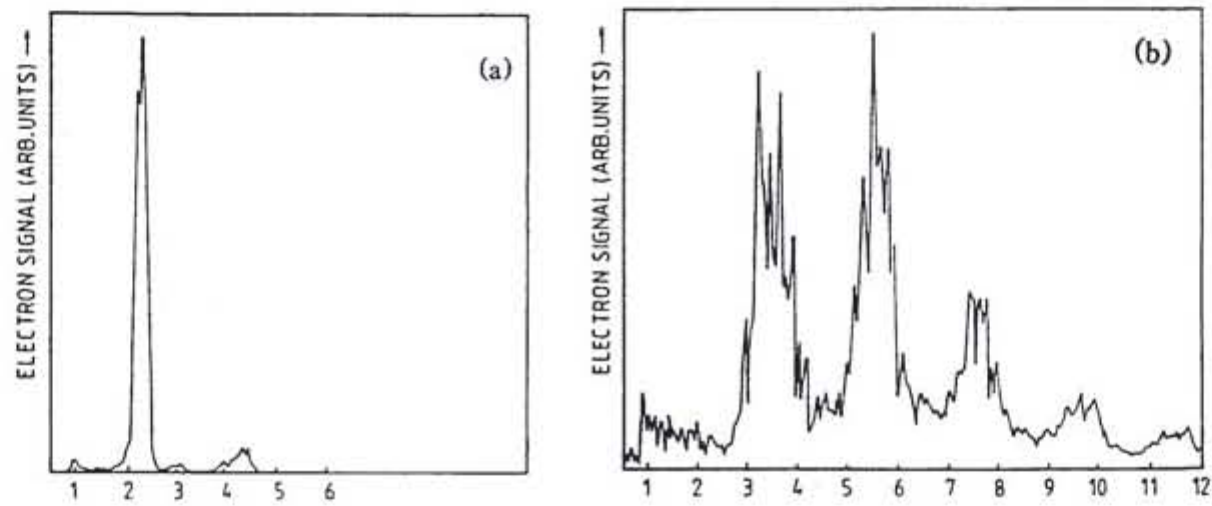

Energie de l'électron (eV)

Figure 33 : Spectre d'énergie des électrons mesuré après une ionisation multiphotonique du Xénon avec une impulsion de $100 \mathrm{fs}$ de durée à $620 \mathrm{~nm}$. La figure (a) est pour une énergie de $11 \mu \mathrm{J}$ par impulsion et la figure (b) pour $29 \mu$ J. D'après Muller et al. (1988). Droits réservés.

Dans le cas d'une ionisation multiple séquentielle, on s'attend à une superposition de spectres d'IAS correspondant aux différentes étapes du processus. La signature d'un processus d'ionisation multiple directe est au contraire différente puisque les électrons émis se partagent l'énergie disponible de façon arbitraire, ce qui doit résulter en un spectre continu.

\subsubsection{Explosion coulombienne et effets nucléaires}

La charge importante et quasi instantanée des atomes fortement ionisés par une impulsion laser femtoseconde super-intense peut entraîner l'explosion de la molécule. Ces expériences maintenant réalisables entraînent et stimulent des travaux théoriques qui prédisent des effets 
inattendus. Cependant il manque aux expériences quelques ordres de grandeur pour observer ces phénomènes.

Des auteurs ont imaginé que les durées de vie radioactive (surtout pour les rayonnements $\beta^{+}$ et $\beta^{-}$) pourraient être perturbées (et peut-être diminuées de deux ordres de grandeur) par les champs électromagnétiques intenses, créés par une impulsion ultracourte. De nombreuses controverses existent dans ce dernier domaine compte tenu d'éventuelles erreurs faites dans l'évaluation des énergies nécessaires pour une telle interaction.

\section{Pllasmas . SOURCES X . ACCÉlÉration DE PARTICUlES}

\subsection{Formation des plasmas}

L'interaction d'une impulsion laser ultra-courte avec la matière solide permet un chauffage direct et rapide du milieu à des densités de l'ordre du $\mathrm{g} / \mathrm{cm}^{3}$, sans expansion hydrodynamique significative (en dessous de $10^{16} \mathrm{~W} / \mathrm{cm}^{2}$ ). L'absorption de l'énergie incidente sur une cible solide en aluminium se fait avec un rendement de $40 \%$ dans l'épaisseur de peau du métal (environ $100 \mathrm{~nm}$ ), d'où la création d'un plasma de température élevée.

Regardons d'abord le cas où l'impulsion laser est relativement longue. Le laser est absorbé jusqu'à la densité critique, atteinte lorsque la fréquence du laser est égale à la fréquence plasma $\left(\omega_{\mathrm{p}}=4 \pi \mathrm{N}_{\mathrm{c}} \mathrm{e}^{2} / \mathrm{m}\right.$, avec $\mathrm{N}_{\mathrm{c}}$ voisin de $\left.10^{2} \mathrm{~cm}^{-3}\right)$. La surface de densité critique forme un miroir réfléchissant qui a le temps de s'éloigner du métal par détente du plasma. Il y a peu de pénétration de la lumière et l'interaction laser-matière se fait avec un milieu de densité faible. Une des conséquences sera que le refroidissement post-impulsion sera lent.

Si l'excitation se fait avec une impulsion brève, l'hydrodynamique du plasma est quasiment figée et le transport vers l'intérieur est modifié par un gradient raide. La faible durée de l'impulsion permet de garder une interface à front raide entre la cible et le vide, ce qui change considérablement les conditions de l'interaction puisque l'onde évanescente dans le solide joue alors un rôle dominant dans le dépôt d'énergie, à des densités supérieures à la densité critique du plasma formé. Le laser interagit avec un milieu dense $\left(10^{23} \mathrm{~cm}^{-3}\right)$. Comme l'épaisseur chauffée est faible, le refroidissement sera rapide. Des mesures par ombroscopie et strioscopie ont montré que le plasma ne s'éloigne pas de plus de $1 \mathrm{~mm}$ (limite de résolution) (Benattar R. et al, 1982).

Sur le plan fondamental, linteraction à haut flux sur un gradient raide de densité électronique pose de nouveaux problèmes théoriques en Physique des plasmas, notamment pour décrire l'absorption du laser et le transport thermique vers l'intérieur de la cible. La zone de dépôt d'énergie laser et la zone d'ablation étant pratiquement confondues, il existe un couplage fort entre les mécanismes d'absorption et de transport.

\subsection{Expansion du plasma}

Le laser est réfléchi par la surface de densité critique (au delà, il y a quand même l'onde évanescente absorbée sur l'épaisseur de peau). La vitesse d'expansion du plasma peut donc être mesurée en regardant l'effet Doppler sur la partie réfléchie du laser.

Deux forces agissent sur le plasma : le gradient thermique qui le pousse à l'expansion et la discontinuité du potentiel pondéromoteur qui va dans le sens d'une compression. En effet l'électron se dirige toujours vers la région où l'intensité lumineuse est la plus faible, c'est à dire celle où le potentiel est le plus bas à cause du terme pondéromoteur. Le plasma voit un champ électromagnétique quasi nul à l'intérieur, dans la région où le laser pénètre peu, et un fort champ à l'extérieur, là où l'onde lumineuse est incidente. L'électron préfere l'intérieur. Le bilan de ces deux forces de sens contraire est que le plasma a un front qui se déplace peu. G. Mourou a 
montré qu'il fallait tenir compte du terme de potentiel pondéromoteur si on voulait expliquer les résultats expérimentaux.

Les propriétés particulières de l'interaction lumière-matière avec des impulsions femtosecondes par rapport à ce qui se passe avec des impulsions nanosecondes font envisager d'utiliser les premières dans le domaine de la fusion nucléaire. Le premier usage serait pour les diagnostiques. Il est aussi envisagé d'utiliser les impulsions femtosecondes comme l'allumette qui déclencherait le processus de fusion nucléaire contrôlée dans le plasma confiné préparé par une impulsion plus longue.

\subsection{Production des rayons $\mathrm{X}$}

On a vu que l'ionisation multiphotonique permet la génération d'harmoniques de rang élevé pour des atomes de gaz rares soumis à des champs électromagnétiques super-intenses créés par des impulsions lasers très courtes. Cependant dans ces harmoniques, le nombre de photons émis par bande spectrale utile est encore trop faible pour en faire un faisceau excitateur dans l'étude des non-linéarités de l'interaction laser-atomes.

Il existe une autre voie. En effet les hautes intensités atteignables en régime subpicoseconde permettent de créer sur des cibles des plasmas qui deviennent des sources d'impulsions brèves dans le domaine des rayons $\mathrm{X}$.

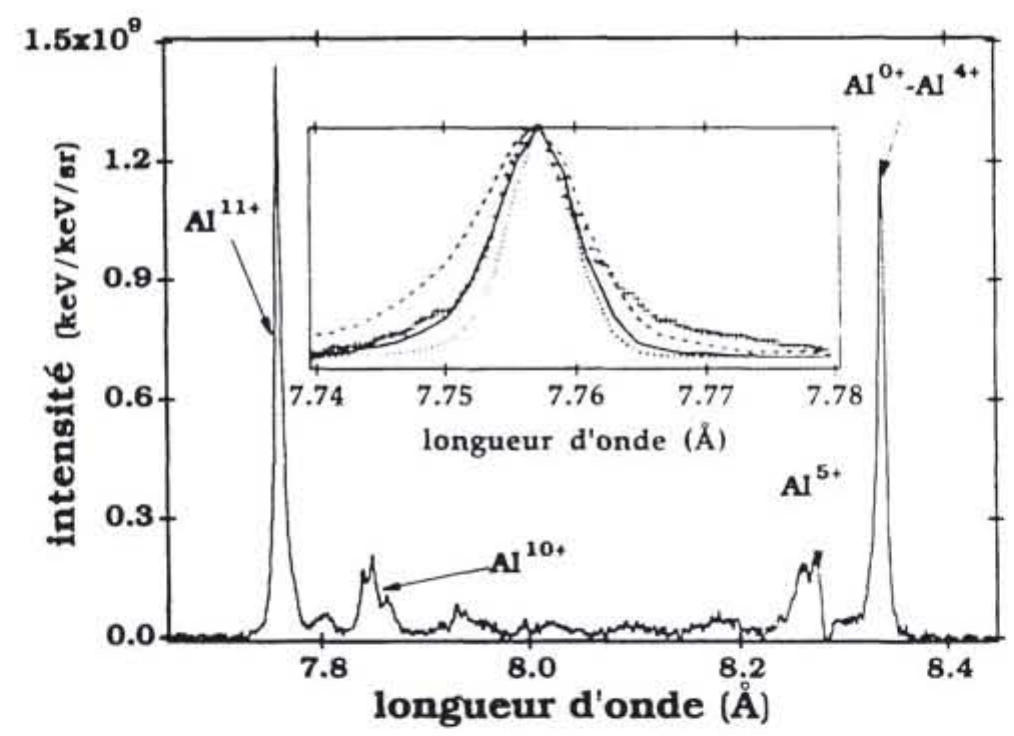

Figure 34 : Spectre de rayonnement X émis par l'aluminium dans la région autour de 8 Angströms. L'encart montre la comparaison entre l'expérience (croix) et un modèle d'effet Stark correspondant à une densité de $5.10^{22}$ (pointillés), $1.10^{23}$ (trait plein) et $2.10^{23} \mathrm{~cm}^{-3}$ (tirets).Daprès Audebert P. et al, 1992. Droits réservés.

Une fois focalisées avec des optiques spécialisées, ces sources donnent des intensités spectrales de l'ordre de $10^{14} \mathrm{~W} / \mathrm{cm}^{2} / \mathrm{eV}$. Celles-ci sont de deux ordres de grandeur supérieures à ce que l'on obtient dans le domaine visible. Une évaluation du rendement (Berreman et al,1990) entre les photons excitateurs et les photons X émis par le plasma se situe entre $5 \%$ et 
$10 \%$. La durée de l'émission des rayons $\mathrm{X}$ a été mesurée par corrélation avec l'impulsion laser femtoseconde et est inférieure à la picoseconde.

Les expériences sur l'aluminium ont été faites sur des cibles formées par une couche mince sur un substrat de silicium. Le spectre détaillé de l'émission de l'aluminium entre 7.7 et $8.5 \AA$ est présenté sur la figure 34 .On observe la raie de $\mathrm{Al}^{11+}$ (héliumoïde), les transitions satellites de $\mathrm{Al}^{10+}$, et les raies $\mathrm{K}_{\alpha}$ dues aux ions, depuis l'état $\mathrm{Al}^{0+}$ (neutre) jusqu'à l'état $\mathrm{Al}^{5+}$ (oxygénö̈de). On devine la présence des ions intermédiaires de $\mathrm{Al}^{6+}$ à $\mathrm{Al}^{9+}$. La comparaison du profil de la raie $\mathrm{Al}^{11}+$ avec les résultats d'un code numérique d'élargissement Stark est présenté dans l'encart. Elle permet de mesurer une densité électronique voisine de $10^{23} \mathrm{~cm}^{-3}$ (légèrement supérieure à la densité des noyaux dans le solide), et une température de l'ordre de cinq millions de degrés Kelvin.

Dans une série d'expériences, on a réduit l'épaisseur de la couche d'aluminium jusqu'à ce que le plasma atteigne le substrat, c'est-à-dire que les raies d'émission de rayons $\mathrm{X}$ du silicium soient observées. Cette émission se produit lorsque l'épaisseur est réduite à environ $1000 \AA$ : cette longueur est une indication de la profondeur de pénétration du front thermique.

Plus à l'intérieur de la cible, la température du silicium mesurée sur les raies $\mathrm{K}_{\alpha}$ est de $150.000^{\circ} \mathrm{K}$. On attribue cette valeur élevée à un chauffage du substrat par des électrons rapides, non thermalisés, provenant du plasma chaud superficiel. Plusieurs mécanismes de transport de l'énergie du laser vers le solide sont ainsi mis en évidence, et particulièrement les mécanismes de transport "délocalisé" tels que le transport par rayonnement, et le transport par les électrons suprathermiques.

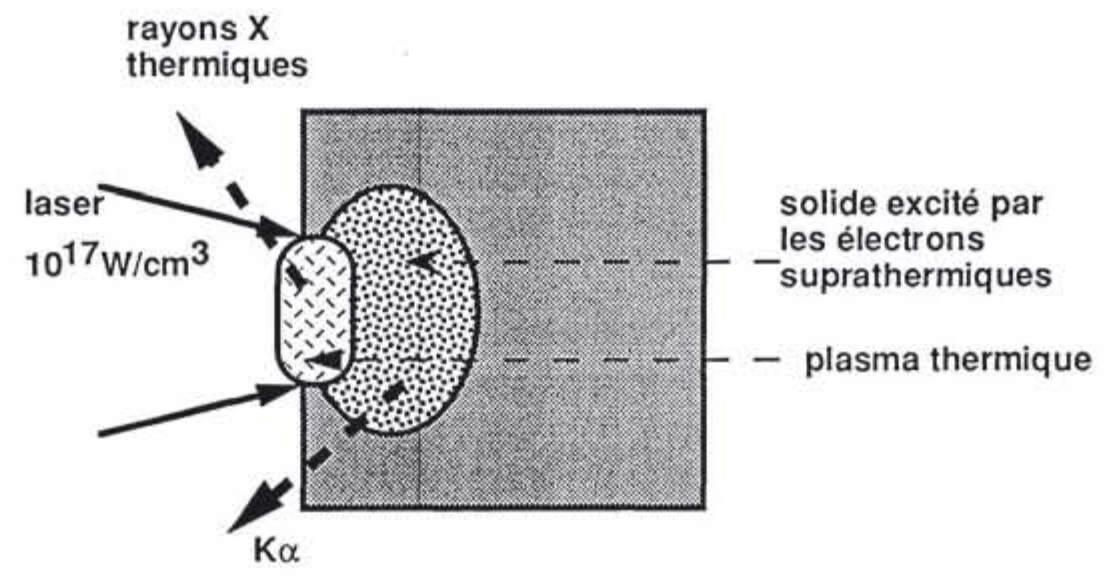

Figure 35 : Représentation schématique des zones excitées directement (par le laser visible) ou indirectement (par les électrons chauds du premier plasma) et émettant des rayons X. La zone du plasma thermique à une épaisseur de l'ordre du dixième de micromètre et une température de $500 \mathrm{eV}$ et la deuxième zone, une épaisseur de 3 micromètre et une température de $20 \mathrm{eV}$.

\subsection{Techniques d'accélération de particules}

Les techniques classiques dans les accélérateurs sont limitées par les claquages des matériaux quand le champ électrique accélérateur devient trop intense. Cette limitation peut être repoussée de plusieurs ordres de grandeur si on peut utiliser un plasma comme milieu accélérateur. L'effet utilisé est l'effet de sillage pour des électrons relativistes. 
Dans un plasma on peut satisfaire à la condition nécessaire pour accélérer un électron en créant une composante longitudinale du champ avec une vitesse de phase proche de la vitesse de la lumière et avec une amplitude importante. Il suffit dans un plasma d'utiliser des modes propres du champ électromagnétique à la fréquence $\omega_{\mathrm{p}}$.

Cette onde plasma peut être créée par une perturbation extérieure, par exemple un laser. L'impulsion lumineuse agit sur les électrons du plasma par effet pondéromoteur. Il y a génération d'une onde plasma de grande amplitude. Le champ électrique lié à ces oscillations peut avoir une vitesse de déplacement (vitesse de phase) voisine de la vitesse des particules injectées dans le plasma (domaine relativiste). Celles-ci resteront donc longtemps dans une zone accélératrice. La vitesse de phase de l'onde plasma est égale à la vitesse de groupe de l'onde électromagnétique qui est ici très proche de la vitesse de la lumière. L'optimisation de cet effet accélérateur consiste à avoir des impulsions laser dont la durée correspondra à une demi-période d'oscillation du plasma.

\subsection{Création de paire électron-positron}

A la fin de cette partie sur les phénomènes en champ laser très intense, il n'est pas inutile de rappeler quelques ordres de grandeur pour des intensités déjà obtenues avec des impulsions de 100 femtosecondes. Les trois premières colonnes s'appliquent au champ lumineux, l'énergie d'oscillation est celle de l'électron dans le champ de radiation.

$\begin{array}{llll}\begin{array}{l}\text { Intensité } \\ \text { Watt } / \mathrm{cm}^{2}\end{array} & \begin{array}{l}\text { Champ électrique } \\ \text { Volt } / \mathrm{cm}\end{array} & \begin{array}{l}\text { Densité d'énergie } \\ \text { Joule } / \mathrm{cm}^{3}\end{array} & \begin{array}{l}\text { Energie d'oscillation } \\ \mathrm{KeV}\end{array} \\ 10^{15} & 9.10^{8} & 3.10^{4} & 0.080 \\ 10^{17} & 9.10^{9} & 3.10^{6} & 8 \\ 10^{19} & 9.10^{10} & 3.10^{8} & 800\end{array}$

Ces chiffres font rêver car ils dépassent ceux auxquels nous sommes habitués.

La loi d'Einstein pour la conservation globale matière-énergie :

$$
\mathrm{E}=\mathrm{m} \mathrm{c}^{2}
$$

donne une énergie de $500 \mathrm{KeV}$ si $m$ est la masse de l'électron. Si on ne tient compte que de l'équation ci-dessus, il semble possible de créer de la matière, en l'occurrence une paire électron-positron pour la conservation de la neutralité, avec des faisceaux lumineux dont l'intensité est de quelques $10^{19} \mathrm{Wcm}^{-2}$. Hélas, dès que les conditions expérimentales semblent se rapprocher d'une limite théorique, un examen plus approfondi de la question repousse cette limite. Il est maintenant prédit que la création d'une paire dans la vide sera fera au-delà de $10^{30} \mathrm{Wcm}^{-2}$ à cause de la conservation d'autres grandeurs. Par contre, au voisinage d'un noyau lourd, certaines contraintes peuvent être levées et la prédiction tourne plutôt autour de $10^{23} \mathrm{Wcm}^{-2}$, ce qu'il n'est pas déraisonnable d'espérer réaliser dans le futur. A vos lasers!

\section{Remerciements}

Je souhaite remercier A. Antonetti, P. Audebert, J.L. Martin, A. Migus et A. Mysyrowicz pour le prêt de leurs documents. 


\section{Bibliographie}

[1] Agostini P., Fabre F., Mainfray G., Petite G., et Rahman N., Phys. Rev. Lett. 42 (1979) 1127

[2] Agostini P., Kupersztych J., Lompré L.A., Petite G. and Yergeau F., Phys. Rev. A 36 (1987) 4111.

[3] Alexander M.G.W., Rühle W.W., Sauer R. et Tsang W.T., Appl. Phys. Lett. 55 (1989) 885

[4] Audebert P., Geindre J.P., Gauthier J.C., Mysyrowicz A., Chambaret J.P., and Antonetti A., Europhysics Letters 19 (1992) 189

[5] Auston D. H., Appl. Phys. Lett. 26, (1975) 101

[6] Balcou Ph., Salières P., and L'Huillier A. , Super Intense Laser-Atom Physics, Piraux et al, (Plenum Press, New York, 1993) pp 9-17

[7] Balcou P. Thèse : Étude de la génération d'harmoniques d'ordre élevé en champ intense. Univ Paris VI (1993)

[8] Bastard G. and Brum J.A., IEEE.J.Quant. Electr. QE 22 (1986) 1625

[9] Benattar R., Geindre J.P., Audebert P., Gauthier J.C., Mysyrowicz A., Chambaret J.P., et Antonetti A., Optics Com. 88 (1982) 376.

[10] Berreman D.W., Bjorkholm J.E., Eichner L., Freeman R.R., Jewell T.E., Mansfield W.M., MacDowell A.A., O'Malley M.L., Raab E.L., Silvast W.T., Szeto L.H., Tennant D.M., Waskiewicz W.K., White D.L., Windt D.L., Wood II.O.R and Bruning J.H., Opt. Lett. 15, (1990) 529

[11] Brum J.A. et Bastard G., Phys. Rev. B 33 (1986), 1421

[12] Deveaud D., Shah J., Damen T.C. et Tsang W.T., Appl. Phys. Lett. 52 (1988) 1886.

[13] Downer M.C., Fork R.L. et Shank C.V., J. Opt. Soc. Am. B 2 (1985) 595

[14] Foing J.P., Hulin D., Joffre M., Jackson M.K., Oudar J.L., Tanguy C. et Combescot M., Phys. Rev. Lett. 68 (1992) 110

[15] Fork R.L., Brito Cruz C.H., Becker P.C. et Shank C.V., Opt. Lett. 12 (1987) 483

[16] Frankel M.Y., Gupta S., Valdmanis J.A. et Mourou G.A., Theory Tech. 39 (1991) 911

[17] Freeman R.R., McIlrath T.J., Bucksbaum P.H. et Bashkansky, Phys. Rev. Lett. 57 (1986) 3156

[18] Freeman R.R., Bucksbaum P.H., Milchberg S., Darack S., Schumacher D., et Geusic M.E., Phys. Rev. Lett. 59 (1987) 1092.

[19] Gauduel Y., Pommeret S., Migus A. et Antonetti A., J. Phys. Chem. 93, (1989) 3880

[20] Grischkowsky D.R., Ketchen M.B., Chi C.C., Duling III I.N., Halas N.J., Halbout J.M. et May P.G., IEEE J. of Quantum Electr. 24, 221 (1988)

[21] Gupta S., Frankel M.Y., Valdmanis J.A., Whitaker J.F., Mourou G.A., Smith F.W. et Calawa A.R., Appl. Phys, Lett. 59 (1991) 3276-3278

[22] Hulin D., Migus A., Antonetti A., Ledoux. I., Badan J., Oudar J.L. et Zyss J., J. Appl. Phys. 64 (1988) 3309-3311

[23] Jackson M.K., Frankel M.Y., Whitaker J.F., Mourou G.A., Hulin D., Antonetti A., Crozat P. et Hafdallah H., Appl. Phys. Lett. 61(1992) 1187.

[24] Lambsdorff M., Kuhl J., Rosenzweig J., Axmann A. et Schneider J., Appl. Phys. Lett. 58 (1991) 1881.

[25] Lee Chi. L., Appl. Phys. Lett. 30, 84 (1977)

[26] Liu Q., Wang J.K. et Zewail A.H., Nature 364 (1993) 427

[27] Martin J.L., Migus A., Poyart C., Le Carpentier Y., Astier R. et Antonetti A., Proc. Natl. Acad Sci. 80 (USA 1983) pp 173-177

[28] Mourchid A., Vanderhagen R., Hulin D. et Fauchet P., Phys. Rev, B 42 (1990) 7667

[29] Muller H.G., Van Linden van den Heuvell H.B., Agostini P., Petite G., Antonetti.A., Franco M., and Migus A. , Phys. Rev. Lett. 60 (1988) 565

[30] Oudar J.L., Hulin D., Migus A., Antonetti A. et Alexandre F , Phys. Rev. Lett. 55 (1985) 2074.

[31] Paye J. et Hulin D., Appl. Phys. Lett. 62 (1993) 1326

[32] Roskoss H.G., Nuss M.C., Shah J., Leo K., Miller D.A.B., Fox A.M., Schmitt-Rink et Köhler K., Phys. Rev. Lett. 68 (1992) 2216 
[33] Tanguy C., Deveaud B., Regreny A., Hulin D. et Antonetti A., Appl. Phys. Lett. 58 (1991) 1283

[34] Valdmanis J.A. et Mourou G.A., IEEE J. Quantum Electr. QE 22 (1986) 69

[35] Valdmanis J.A., Electron. Lett. 23, 1308 (1987)

[36] Valdmanis J.A., Semiconductors and semimetals 28, (Academic Press Inc. 1990), p 135

[37] von der Linde D., Kuhl J. et Klingenberg H., Phys. Rev. Lett. 44 (1980) 1505

[38] Vos M.H., Rappaport F., Lambry J.C., Breton J. et Martin J.L., Nature (London) 363, (1993) 320-325

[39] Young J.F., Proc. Int. Quantum Electr. Conf. (Anaheim USA, 1994)

[40] Zewail A.H., Femtosecond Reaction Dynamics, (Wiersma D.A. éditeur, North-Holland Amsterdam 1994) pp 1-28 